\title{
Test of an additive diquark spectator model for meson-baryon quasi-two-body reactions
}

\author{
M. Zralek,* W.J. Metzger, and R.T. Van de Walle \\ Fysisch Laboratorium Universiteit van Nijmegen, Nijmegen, Netherlands \\ C. Dionisi, A. Gurtu, ${ }^{\dagger}$ and M. Mazzucato \\ CERN, European Organization for Nuclear Research, Geneva, Switzerland \\ B. Foster \\ Nuclear Physics Laboratory, University of Oxford, Oxford, United Kingdom \\ (Received 12 June 1978)
}

\begin{abstract}
An additive diquark model is proposed to relate the forward cross sections of meson-baryon quasi-two-body reactions. The model consists of a quark-diquark structure for baryons combined with additivity of quark(di)quark amplitudes. Good agreement is found between this model, which contains only one free parameter, and a large body of experimental data for charge-exchange and strangeness-exchange meson-baryon twobody reactions. This is in contrast to the SU(6)-symmetric additive quark model which disagrees violently with the data.
\end{abstract}

\section{INTRODUCTION}

The successes of the so-called naive (nonrelativistic) quark model are well known. In this model mesons are bound $q \bar{q}$ states while baryons are three-body bound states of the type $q q q$. With this model many features of hadron spectroscopy can be understood. For baryon states there has been a problem for quite some time with quark statistics; the best agreement with the experimental data was obtained with the assumption of an SU(6)-symmetric $s$-wave $\left(56,0^{+}\right)$ground state and required the awkward assumption that quarks were parafermions of order three (i.e., three spin- $\frac{1}{2}$ particles effectively obeying Bose statistics). This problem is now generally felt resolved by the hypothesis that quarks carry an additional quantum number (color) and that baryons are color singlets. Problems which still remain in baryon spectroscopy are the undetected 20 multiplets and the dynamic reasons for the pattern $\left(56,0^{+}\right),\left(70,1^{-}\right),\left(56,2^{+}\right)$, etc. Experimentally the 20 is difficult to detect, since it does not couple to a $0^{-}$meson 35 and a $\frac{1+}{2}$ baryon 56 , but the quantity of data on baryonmeson-meson systems has now become sufficiently large to worry us.

The success of the quark model has not been limited to spectroscopy and the correlation of static hadron properties such as mass splittings, magnetic moments, etc. For many years the quark model has been shown to be an important tool for correlating weak, electromagnetic, and strong decays of hadrons and for producing relations between total cross sections (or forward elastic scattering) and between peripheral quasitwo-body scattering processes. In the latter case both cross section and polarization (spin density matrix elements or statistical tensors) predictions appeared over and over again to be in agreement with the data. However, there also have been a few persistent problems in the application of the quark model to scattering processes and here too these complications arose in the quarkmodel interpretation of baryons. Well known in this respect are the erroneous prediction by $\mathrm{SU}(6)$ of the ratio between the $n$ and $p$ deep-inelastic structure functions as measured by $e$ and $\nu$ scattering ${ }^{1}$ and the inability of $\mathrm{SU}(6)$ to predict correctly the nucleon $G_{A} / G_{V}$ ratio, the ratio of the axial-vector to vector coupling constants for the nucleon in $\beta$ decay. ${ }^{2}$ Of probably even more direct interest for the symmetric quark model are the discrepancies observed for the forward cross sections of the reactions

$$
\begin{aligned}
& K^{-} p \rightarrow M^{0}+\left(\Lambda^{0}, \aleph^{0}, Y_{1385}^{* 0}\right) \\
& \left(M^{0}=\text { a neutral nonstrange meson }\right) .
\end{aligned}
$$

The serious disagreement observed for these ratios between the quark-model predictions and the experimental data was first discussed by Hirsch et al. ${ }^{3}$ Recently our collaboration, using data of a large $K^{-} p$ experiment $(\sim 130$ events $/ \mu \mathrm{b})$ as well as data from a variety of other experiments, has found that this effect occurs not only systematically in the cross sections integrated over the forward hemisphere but is also consistently present at all forward $t$ in the differential cross sections. ${ }^{4}$ On the other hand, the polarizations of the baryons for natural-parity exchange were found to be in agreement with the SU(6) predictions, consistent with the assumption that the two spectator quarks of the proton were 
in the same pure spin-isospin state as in the $\mathrm{SU}(6)$ proton wave function.

This disagreement in the cross sections for reactions (1) should be contrasted with the good agreement with the additive-quark-model predictions found for reactions such as $^{2,5,6}$

$$
\begin{aligned}
& K^{-} p \rightarrow\left(\rho^{0}, \omega, \phi\right)+B^{0} \\
& \left(B^{0}=\text { a neutral strange baryon }\right) .
\end{aligned}
$$

It should be stressed that the predictions for reactions (1) test the specific form of the baryon form factor and/or wave function while those connected with reactions (2) follow from quark-model selection rules only.

To remedy these baryon defects (while keeping a baryon made up of three quarks) one can invoke essentially two "solutions" (or rather, groups of solutions):

(a) One can postulate a breakdown of the additive reaction model (e.g. by introducing double $q$ scattering, etc.).

(b) One can break the SU(6) form factors that appear in the symmetric quark model.

The first possibility raises the question: Why should this breakdown only occur in reactions (1) (and e.g. not contribute to exotic exchanges)?

The second approach has two subvariants:

(b1) One can assume special reaction mechanisms fav̀oring certain strangeness-exchange transitions over other ones.

(b2) One can assume that the wave function of the baryons involved breaks $\mathrm{SU}(6)$.

The first variant-in the absence of a satisfactory way of calculating these special reaction mechanisms-remains an ad hoc assumption with little or no predictive power outside the direct cases considered. Most of the suggestions found in the literature have therefore centered around approach (b2).

Also within (b2) there are several possible roads:

(b2a) One can stay within SU(6) and consider changes in the form factor resulting from configuration mixing in the baryon wave functions.

(b2b) One can break SU(6) and consider approximately $\mathrm{SU}(6)$-symmetric baryon wave functions.

(b2c) One can break SU(6) by starting from a wave function resulting from a (nonsymmetric) substructure model for the three quarks inside the baryon.

Possibility (b2a) has been suggested by Hirsch et $a l .{ }^{7}$ They estimated that mixing with members of an $L=2 J^{P}=\frac{1}{2}^{+}$decuplet (having total quark spin $=\frac{3}{2}$ ) could affect the $\Sigma$ and $Y^{*}$ cross sections by an amount sufficiently large to explain the discrepancy. However, detailed calculations of these mixing effects are not possible without a specific model for the transition matrix between the $L=0$ and $L=2$ configurations and a knowledge of the (differing) spatial part of the wave function for these two states.

An example of the approach (b2b) is the group $\mathrm{SU}(3) \times \mathrm{SU}(2)$. This group contains three parameters for which a particular set of values again yields the $S U(6)$ baryon wave functions. Since there are no well-established baryon states belonging to the 20 and $\underline{70_{2}}$ representations of $\mathrm{SU}(6)$, it seems reasonable to fix two of the parameters such that these states also do not occur in SU(3) $\times \mathrm{SU}(2)$. There remains then one free parameter. It turns out, however, that the cross-section ratios of reaction (1) are independent of this parameter. ${ }^{8}$

In this paper we will examine one of the possibilities of approach (b2c). Our first task is then to make a specific assumption for the baryon substructure. We will use a model already discussed in the literature: the so-called diquark model. We will use this model not only to explain the discrepancies observed in reactions (1) but also to predict new relations for another group of reactions that are sensitive to possible baryon substructure [namely, the charge-exchange reactions of the type

$\pi^{-} p \rightarrow M^{0}+\left(n, \Delta^{0}\right)$ and $\left.K^{-, 0} p \rightarrow K^{0,+}\left(K^{* 0,+}\right)+\left(n, \Delta^{0}\right)\right]$.

This model assumes that the baryon is a bound state of a (more tightly bound) two-quark system (the diquark) and a third quark. This reduction of the baryon to a two-body structure is really the next simplest thing to the situation of a completely symmetric three-quark state. The diquark model was first proposed by Lichtenberg and Tassie ${ }^{9}$ several years ago essentially to explain static hadron properties. In Sec. II we will discuss in detail this model and its assumptions as we have put it to use for strangeness - and charge-exchange meson-baryon reactions. However, we would like to make two remarks here:

(1) Our diquark model respects $\mathrm{SU}(6)$ for the diquark structure but breaks $\mathrm{SU}(6)$ through the coupling of the diquark with the third quark. This starting point of the model does not imply that the total wave function cannot be approximately SU(6)invariant; on the contrary, as we will indicate later, the wave function resulting from the diquark model contains a single parameter $(\Gamma)$ which in a certain limit makes the wave functions (analytically) identical to those predicted by the symmet- 
ric three-quark model. The essential difference with approach (b2b) is that there the wave function is symmetric (in the three quarks) for all values of the parameters involved; in the diquark model it is symmetric for only one specific $\Gamma$ value.

(2) The essence of the diquark model as used by us in this paper is that one avoids the necessity of complete three-quark symmetrization by treating the diquark as an "object" distinguishable from the remaining quark. This does not necessarily imply that diquarks have to exist as elementary objects themselves. Justification for not symmetrizing could also come from the spatial part of the wave function, which is unspecified in our model. If one quark is spatially far removed from the other two, the effect of symmetrization is practically nil; the diquark assumption could then be an approximation (in terms of symmetry requirements) for the correct three-quark dynamics inside the baryon, an approximation useful to calculate the approximate $\mathrm{SU}(6)$ behavior of the baryon but to be explained later in a more fundamental way. In the context of our paper the word "diquark" never has to mean more than a (sufficient) clustering of two quarks inside a baryon.

Lichtenberg and co-workers have used the diquark model to calculate the electromagnetic and medium-strong mass splitting of baryons and their magnetic moments. ${ }^{10}$ In terms of establishing the superiority of the diquark model over the unbroken $\mathrm{SU}(6)$ model, their findings were inconclusive. Most of the static diquark predictions worked just as well (or just as badly) as the corresponding $\mathrm{SU}(6)$ ones. Where differences were predicted the experimental errors were not small enough to allow differentiation.

\section{THE ASSUMPTIONS OF THE DIQUARK SPECTATOR MODEL}

There are essentially five assumptions that will be used in deriving experimentally verifiable relations from the quark-diquark model hypothesis. We will discuss them one by one; we will enumerate all our assumptions (even some of the more trivial ones) in order to present a complete picture.

(a) First and foremost the diquark spectator model use the additivity assumption. ${ }^{11}$ As in the conventional additive quark model this implies usage of an amplitude for a quasi-two-body interaction (with momentum transfer $t$ ) of the form

$$
H_{a}(t)=D(t) \sum_{a^{\prime}} C_{a^{a}}^{a^{\prime}} h_{a^{\prime}}(t),
$$

where $a, a^{\prime}$ represent the helicity or transversity configuration of the particles and quarks (or diquarks), respectively, $h_{a}(t)$ represents the quark-quark, quark-diquark, or diquark-diquark amplitudes, $C_{a}^{a^{\prime}}$ are the coefficients (weight factors) derived from the particle wave functions (as postulated by the model), and $D(t)$ is a form factor which effectively takes into account that the spectator diquark or quark must be dragged away from the collinear (incident) direction by the quark or diquark undergoing the interaction.

(b) The second important assumption concerns the quark structure of the hadrons and the wave function resulting from it. For mesons we stay within the conventional $q \bar{q}$ structure. For baryons, however, we will assume that-as a result of the $q-q$ interactions - they consist of two quarks forming a more or less bound state, the diquark, which in turn interacts with a third quark thus forming a baryon. We shall assume that a diquark has the same quantum number as a bound state of two quarks with zero orbital angular momentum. We treat the diquark as being "elementary" only in the sense that when combining it into an $s$ state with a quark to form the baryon, we do not symmetrize the wave function in the added third quark with respect to the two quarks already present in the diquark. It should be emphasized that for ground-state baryons (with all three quarks in relative $s$ states) the above structure assumption is equivalent to the assumption of the presence of a repulsive three-body component in the interquark force.

Making the usual assumption that quarks belong to the fundamental six-dimensional representation of $\mathrm{SU}(6)$, the $\mathrm{SU}(6)$ multiplet of the diquark must be given by

$$
\underline{6} \times \underline{6}=\underline{21}+\underline{15} \text {. }
$$

The most specific-and in that sense the most important-hypothesis concerning the diquark model is that the 15-dimensional diquark (if indeed bound at all) lies much higher in energy than the 21. Then the baryon can only result from the combination of the 21 diquark with a third quark as follows:

$\underline{21} \times \underline{6}=\underline{56}+\underline{70}$.

The assumption that the diquark belongs only to the 21 therefore explains why all low-lying baryons belong (approximately) either to a 56- or 70-dimensional representation of $\mathrm{SU}(6)$ and in particular why the 20-plet should be absent. ${ }^{12}$ Furthermore, with the orbital angular momentum assumptions already made (and the assumption that the relative intrinsic parity of the quark and diquark is positive) we immediately have the rule that $P_{\text {baryon }}=(-1)^{L}$ where $L$ is the relative orbital angular momentum of the quark-diquark system. 
TABLE I. Quantum numbers of diquarks.

\begin{tabular}{|c|c|c|c|c|c|c|}
\hline & $I$ & $I_{z}$ & $Y$ & $Q$ & Spin & $\begin{array}{l}\text { Quark } \\
\text { content }\end{array}$ \\
\hline$s_{1}$ & 1 & +1 & $\frac{2}{3}$ & $\frac{4}{3}$ & 1 & $\mathbb{P P}$ \\
\hline$s_{2}$ & 1 & 0 & $\frac{2}{3}$ & $\frac{1}{3}$ & 1 & $\mathbb{P} \mathscr{X}$ \\
\hline$s_{3}$ & 1 & -1 & $\frac{2}{3}$ & $-\frac{2}{3}$ & 1 & $\operatorname{sex}$ \\
\hline$s_{4}$ & $\frac{1}{2}$ & $\frac{1}{2}$ & $-\frac{1}{3}$ & $\frac{1}{3}$ & 1 & $P \lambda$ \\
\hline$s_{5}$ & $\frac{1}{2}$ & $-\frac{1}{2}$ & $-\frac{1}{3}$ & $-\frac{2}{3}$ & 1 & $\mathscr{N \lambda}$ \\
\hline$s_{6}$ & 0 & 0 & $-\frac{4}{3}$ & $-\frac{2}{3}$ & 1 & $\lambda \lambda$ \\
\hline$t_{1}$ & 0 & 0 & $\frac{2}{3}$ & $\frac{1}{3}$ & 0 & $P \mathscr{H}$ \\
\hline$t_{2}$ & $\frac{1}{2}$ & $\frac{1}{2}$ & $-\frac{1}{3}$ & $\frac{1}{3}$ & 0 & $P \lambda$ \\
\hline$t_{3}$ & $\frac{1}{2}$ & $-\frac{1}{2}$ & $-\frac{1}{3}$ & $-\frac{2}{3}$ & 0 & $\mathscr{T \lambda}$ \\
\hline
\end{tabular}

This explains the occurrence of only $0^{+}, 1^{-}, 2^{+}$, etc. SU(6) multiplets. With the additional assumption of a sizable exchange force between quark and diquark, only the so-called minimal spectrum $\left(56, L_{\text {even }}^{+}\right)$and $\left(70, L_{\text {odd }}^{-}\right)$would occur, in agreement with experiment. ${ }^{12}$ We will of course adopt the experimentally favored (approximate) assignment of the nucleon-octet and the $\Delta$ decuplet to the $\left(56,0^{+}\right)$representation of $\mathrm{SU}(6)$.

The SU(3) content of the 21-dimensional diquark is an $\mathrm{SU}(3)$ sextet of spin 1 and an SU(3) triplet of spin 0 . We shall refer to these diquarks as sextets and triplets respectively (symbols $s$ and $t)$; in Table I we present a summary of their SU(3) and SU(2) quantum numbers. ${ }^{13}$ Note that the 21 plet states are symmetric in the $S U(6)$ variables. Thus the nonstrange diquark satisfies the twonucleon state rule that $I=0$ corresponds to total spin $S=0$ and $I=1$ to $S=1$. Assuming that the diquark wave function is antisymmetric (since quarks are fermions), then since the diquark must be antisymmetric in color in order that the baryon be fully antisymmetric in color, one finds that the relative orbital angular momentum between the two quarks of the diquark must be even for the 21-plet and odd for the 15-plet. An $l=0$ diquark should thus be in the 21-plet. This may be considered as an additional argument in favor of neglecting the 15 , at least for the lowmass baryons, which are considered in this paper.

With these diquarks we now consider the most general possible (internal) wave functions for the baryons entering into our analysis $\left(p, n, \Delta^{0}, \Lambda^{0}, \Sigma^{0}\right.$, $\left.Y^{*^{0}}\right)$ imposing $\mathrm{SU}(3)$ conservation but allowing SU(6) breaking in the coupling of the diquark to the third quark. ${ }^{13}$ Table II gives the resulting wave functions. The $\mathrm{SU}(6)$ breaking is controlled by an angle $\Gamma$; for $\Gamma=\pi / 4$ they reproduce exactly the unbroken $\mathrm{SU}(6)$ wave functions, i.e., the wave functions of the symmetric three-quark model. For any other value of $\Gamma$ the wave functions in Table II differ from the SU(6) wave functions specifically by the fact that they are symmetrized only in terms of the two quarks inside the diquark and do not contain any symmetry requirements that would result from interchanging the third quark with one of the quarks inside the diquark. Note that the wave functions of decuplet members do not contain $\Gamma$; for the decuplet we cannot break $\mathrm{SU}(6)$ without also breaking SU(3).

(c) We now turn to a discussion of the individual quark-quark and quark-diquark amplitudes as they contribute (within the additivity model) to peripheral meson-baryon scattering in general and the two-body reactions considered here in particular. In the usual quark model we only have to consider quark-quark amplitudes; one then deals with two possible types of processes, namely

TABLE II. Internal baryon wave functions in quark-diquark model [SU(3) conserved; SU(6) broken]. $\alpha_{ \pm}$denote quark up and down spin wave functions. $\chi_{ \pm}$and $\chi_{m}$ denote the combined quark-sextet diquark spin wave functions for a total spin $\frac{1}{2}$ and spin $\frac{3}{2}$, respectively. $q_{1}, q_{2}$ and $q_{3}$ represent the $\mathcal{P}, \mathcal{X}$, and $\lambda$ quarks, respectively. The sign of our $\Sigma$ wave function differs from that of Ref. 13. We have used the sign convention of de Swart (Ref. 14).

$$
\begin{aligned}
& |p, \pm\rangle=\sin \Gamma \frac{1}{\sqrt{3}}\left(\sqrt{2} s_{1} q_{2}-s_{2} q_{1}\right) \chi_{ \pm}+\cos \Gamma t_{1} q_{1} \alpha_{ \pm} \\
& |n, \pm\rangle=\sin \Gamma \frac{1}{\sqrt{3}}\left(s_{2} q_{2}-\sqrt{2} s_{3} q_{1}\right) \chi_{ \pm}+\cos \Gamma t_{1} q_{2} \alpha_{ \pm} \\
& |\Lambda, \pm\rangle=\sin \Gamma \frac{1}{\sqrt{2}}\left(s_{4} q_{2}-s_{5} q_{1}\right) \chi_{ \pm}+\cos \Gamma \frac{1}{\sqrt{6}}\left[\left(t_{2} q_{2}-t_{3} q_{1}\right)+2 t_{1} q_{3}\right] \alpha_{ \pm} \\
& \left|\Sigma^{0}, \pm\right\rangle=-\sin \Gamma \frac{1}{\sqrt{6}}\left[2 s_{2} q_{3}-\left(s_{5} q_{1}+s_{4} q_{2}\right)\right] \chi_{ \pm}-\cos \Gamma \frac{1}{\sqrt{2}}\left(t_{3} q_{1}+t_{2} q_{2}\right) \alpha_{ \pm} \\
& \left|Y^{* 0}, m\right\rangle=\frac{1}{\sqrt{3}}\left(s_{5} q_{1}+s_{4} q_{2}+s_{2} q_{3}\right) \chi_{m} \\
& \left|\Delta^{0}, m\right\rangle=\frac{1}{\sqrt{3}}\left(s_{3} q_{1}+\sqrt{2} s_{2} q_{2}\right) \chi_{m}
\end{aligned}
$$




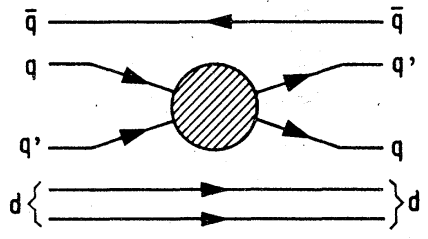

Quark

Rearrangement

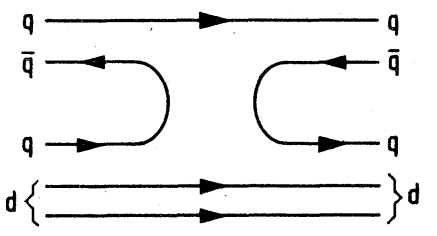

Quark

Annihilation

(b)

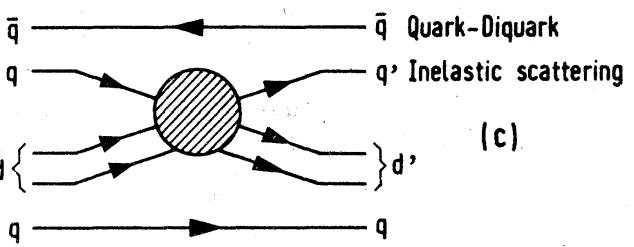

FIG. 1. Quark diagrams for peripheral quasi-twobody scattering: (a) quark rearrangement, (b) quark annihilation-creation, and (c) quark-diquark inelastic scattering.

so-called rearrangement processes and annihilation-creation processes. Figures $1(a)$ and $1(b)$ show these two possibilities as they appear in the quark-diquark model.

We should in principle also consider quarkdiquark amplitudes of the type shown in Fig. 1(c). However, for the two-body processes considered here (i.e., charge- and strangeness-exchange twobody reactions) these amplitudes must be inelastic and of a rather specific type; they require the substitution of one of the quarks inside the diquark by one of the quarks from the meson withoutbreaking the diquark structure. This would presumably be a rather improbable process; normally a quark-diquark scattering would probably either be elastic or else break up the diquark structure. We will therefore neglect all contributions from diagram 1(c). ${ }^{15}$ An additional (heuristic) reason for not including these contributions in our model calculations is that they would substantially increase the number of parameters used in the model; as we will show later, the model does not need these additional parameters.

(d) The final assumption of our model concerns the form factor $D(t)$. We cannot calculate this form factor because we do not know the space part of the wave functions involved. We will make the usual assumption that this space part depends both on the total angular momentum inside the baryon (in our case on $L$, the relative orbital angular momentum between the quark and the diquark) and on the SU(6) representation to which the internal baryon wave function belongs in the limit of unbroken $S U(6)(\Gamma \rightarrow \pi / 4)$, i.e., $D_{L}^{(56)}(t)$, $D_{L}^{(70)}(t)$, etc. This leaves us with just one (common) form factor $D_{0}^{(56)}$ for all of the $\left(56,0^{+}\right)$ baryons considered here.

\section{PREDICTIONS OF THE DIQUARK SPECTATOR MODEL}

We will use the model to predict relations between the peripheral cross sections and polarizations of the strangeness-exchange reactions

$$
A+p \rightarrow M+\left(\Lambda^{0}, \Sigma^{0}, Y_{1385}^{* 0}\right)
$$

and the charge-exchange reactions

$$
A+p \rightarrow M+\left(n, \Delta^{0}\right),
$$

where $A$ and $M$ are mesons satisfying all required conservation laws. We will consider the reactions where $A$ is always a pseudoscalar meson ( $\pi$ or $K$ ) and $M$ is either a pseudoscalar or a vector meson. From now on we will suppress all charge indices on the baryon considered and assume we deal with neutral outgoing particles only $\left(Y=Y_{1385}^{* 0}\right)$; $\sigma(\Lambda), \sigma(\Sigma)$, etc. will be used as an abbreviation for the $\sigma$ (or $d \sigma / d t$ ) of $A p \rightarrow M \Lambda, A p \rightarrow M \Sigma$, etc.

Tables III and IV show the different mesonbaryon amplitudes contributing to the strangeness-exchange and charge-exchange reactions, respectively. All amplitudes are expressed in the transversity frame, i.e., with respect to a spin quantization axis perpendicular to the twobody reaction plane. The symbol $m$ will be used as a general index to indicate quantum numbers along this axis. In the tables we not only distinguish the case of a final-state pseudoscalar meson and vector meson, but for the latter we also distinguish between the three possible "transversities" with which it can be produced $(m=0, \pm 1)$. There exists a correlation between the naturality of the particles exchanged in a two-body process and the transversity state of the meson in the final state. ${ }^{16}$ If the produced meson is a pseudoscalar $\left(M=0^{-}\right)$or a vector meson $\left(M=1^{-}\right)$with $m=0$, the exchanged particle has natural parity $(N)$; for the case $M=1^{-}$with $m= \pm 1$ the exchanged object must have unnatural parity $(U)$. (Actually, for $M=1^{-}$these statements are strictly speaking valid only in the limit $s \rightarrow \infty$.)

The amplitudes are expressed in terms of $x=\sin ^{2} \Gamma$ [where $\Gamma$ is the $S U(6)$-breaking angle 
TABLE III. Meson-baryon transversity amplitudes for strangeness-exchange reactions (in terms of $x=\sin ^{2} \Gamma$ and meson-quark amplitudes $P_{i}, V_{i}$; see text for definitions).

\begin{tabular}{|c|c|c|c|c|}
\hline \multirow{2}{*}{$\begin{array}{l}\text { Reactions } \\
\text { Amplitudes }\end{array}$} & \multirow{2}{*}{$\begin{array}{c}0^{-}+p \rightarrow 0^{-}+(\Lambda, \Sigma, Y) \\
(\mathrm{NP} \text { exch })\end{array}$} & \multicolumn{3}{|c|}{$0^{-}+p \rightarrow 1^{-}+(\Lambda, \Sigma, Y)$} \\
\hline & & $\begin{array}{c}m=0 \\
\text { (NP exch) }\end{array}$ & $\begin{array}{c}m=+1 \\
\text { (UP exch) }\end{array}$ & $\begin{array}{c}m=-1 \\
\text { (UP exch) }\end{array}$ \\
\hline$p_{1 / 2} \rightarrow \Lambda_{1} q_{2}$ & $\frac{\sqrt{2}}{\sqrt{3}}(1-x) P_{1}$ & $\frac{\sqrt{2}}{\sqrt{3}}(1-x) V_{1}$ & 0 & 0 \\
\hline$p_{1 / 2} \rightarrow \Lambda_{-1 / 2}^{0}$ & 0 & 0 & $\frac{\sqrt{2}}{\sqrt{3}}(1-x) V_{3}$ & $\frac{\sqrt{2}}{\sqrt{3}}(1-x) V_{5}$ \\
\hline$p_{-1 / 2} \rightarrow \Lambda_{1} \rho_{2}$ & 0 & 0 & $\frac{\sqrt{2}}{\sqrt{3}}(1-x) V_{4}$ & $\frac{\sqrt{2}}{\sqrt{3}}(1-x) V_{6}$ \\
\hline$p_{-1 / 2} \rightarrow \Lambda_{-1 / 2}^{0}$ & $\frac{\sqrt{2}}{\sqrt{3}}(1-x) P_{2}$ & $\frac{\sqrt{2}}{\sqrt{3}}(1-x) V_{2}$ & 0 & 0 \\
\hline$p_{1 / 2} \rightarrow \Sigma_{1} g_{2}$ & $\frac{\sqrt{2}}{9} x\left(2 P_{2}+P_{1}\right)$ & $\frac{\sqrt{2}}{9} \times\left(2 V_{2}+V_{1}\right)$ & $\begin{array}{r}0 \\
-\quad 0\end{array}$ & 0 \\
\hline$p_{1 / 2} \rightarrow \Sigma_{-1 / 2}^{0}$ & 0 & 0 & $-\frac{\sqrt{2}}{9} x V_{3}$ & $-\frac{\sqrt{2}}{9} \times V_{5}$ \\
\hline$p_{-1 / 2} \rightarrow \Sigma_{1 / 2}$ & 0 & 0 & $-\frac{\sqrt{2}}{9} \times V_{4}$ & $-\frac{\sqrt{2}}{9} \times V_{6}$ \\
\hline$p_{-1 / 2} \rightarrow \Sigma_{-1 / 2}^{0}$ & $\frac{\sqrt{2}}{9} x\left(2 P_{1}+P_{2}\right)$ & $\frac{\sqrt{2}}{9} x\left(2 V_{1}+V_{2}\right)$ & 0 & 0 \\
\hline$p_{1 / 2} \rightarrow Y_{3 / 2}^{0}$ & 0 & 0 & $-\frac{\sqrt{2 x}}{3 \sqrt{3}} V_{4}$ & $-\frac{\sqrt{2 x}}{3 \sqrt{3}} V_{6}$ \\
\hline$p_{1 / 2} \rightarrow Y_{1} \oint_{2}$ & $\frac{\sqrt{2 x}}{9}\left(P_{1}-P_{2}\right)$ & $\frac{\sqrt{2 x}}{9}\left(V_{1}-V_{2}\right)$ & 0 & 0 \\
\hline$p_{1 / 2} \rightarrow Y_{-1 / 2}^{0}$ & 0 & 0 & $\frac{\sqrt{2 x}}{9} V_{3}$ & $\frac{\sqrt{2 x}}{9} V_{5}$ \\
\hline$p_{1 / 2} \rightarrow Y_{-3 / 2}^{0}$ & 0 & 0 & 0 & 0 \\
\hline$p_{-1 / 2} \rightarrow Y_{3} q_{2}$ & 0 & 0 & 0 & 0 \\
\hline$p_{-1 / 2} \rightarrow Y_{1} g_{2}$ & 0 & 0 & $-\frac{\sqrt{2 x}}{9} V_{4}$ & $-\frac{\sqrt{2 x}}{9} V_{6}$ \\
\hline$p_{-1 / 2} \rightarrow Y_{-1 / 2}^{0}$ & $\frac{\sqrt{2 x}}{9}\left(P_{1}-P_{2}\right)$ & $\frac{\sqrt{2 x}}{9}\left(V_{1}-V_{2}\right)$ & 0 & 0 \\
\hline$p_{-1 / 2} \rightarrow Y_{-3 / 2}^{0}$ & 0 & 0 & $\frac{\sqrt{2 x}}{3 \sqrt{3}} V_{3}$ & $\frac{\sqrt{2 x}}{3 \sqrt{3}} V_{5}$ \\
\hline
\end{tabular}

previously defined] and the quantities $P_{i}$ and $V_{i}$. In terms of the quantities in Eq. (3), the $x$-dependent factors are derived from the $C_{a}^{a^{\prime}}$ coefficients and the $P_{i}, V_{i}$ are proportional to combinations of the $D(t) h_{a},(t)$. The $P_{i}$ and $V_{i}$ are in fact meson-quark transversity amplitudes; they depend on the meson quark structure and are linear combinations of the quark-quark transversity amplitudes defined by the diagrams Fig. 1(a) and Fig. 1(b). As an example for reactions $K^{-} p \rightarrow \pi^{0}+\cdots \cdot$ we have

$$
\begin{aligned}
P_{1} & =\left\langle\pi^{0} q_{3^{+}} \mid K^{-} q_{1^{+}}\right\rangle \\
& =\frac{1}{2 \sqrt{2}}\left(\left\langle q_{1^{+}} q_{3^{+}} \mid q_{3^{+}} q_{1^{+}}\right\rangle+\left\langle q_{1-} q_{3^{+}} \mid q_{3^{+}} q_{1^{+}}\right\rangle\right)
\end{aligned}
$$

and for reactions $K^{-} p \rightarrow \rho_{m=0}^{0}+\cdots$ we have

$$
\begin{aligned}
V_{1} & =\left\langle\rho_{0^{0}}^{0} q_{3^{+}} \mid K^{-} q_{1^{+}}\right\rangle \\
& =\frac{1}{2 \sqrt{2}}\left(\left\langle q_{1-} q_{3^{+}} \mid q_{3^{-}} q_{1^{+}}\right\rangle-\left\langle q_{1^{+}} q_{3^{+}} \mid q_{3^{+}} q_{1^{+}}\right\rangle\right)
\end{aligned}
$$

Note that although we have formulated the mesonquark amplitudes in terms of a particular mesonquark structure and a particular meson-quark interaction mechanism, our results will be independent of these details and will depend only on the assumptions made at the baryon vertex.

We are now ready to extract predictions from the model by eliminating the unknown amplitudes $P_{i}, V_{i}\left(P_{i}^{\prime}, V_{i}^{\prime}\right)$. We will write all our predictions in terms of cross sections corrected for kinematic effects resulting from mass differences and use the symbol $\bar{\sigma}$ to indicate these.

Some of the relations obtained do not depend on $x$; they are relations which our model has in common with unbroken SU(6). Within our model the validity of these relations is coupled to the assumption that we can neglect the quark-diquark inelastic amplitudes. As the aim of this paper is to explain disagreements with SU(6) predictions in situations where the diquark model does yield predictions different from unbroken $\mathrm{SU}(6)$, we 
TABLE IV. Meson-baryon transversity amplitudes for charge-exchange reactions (in terms of $x=\sin ^{2} \Gamma$ and mesonquark amplitudes $\boldsymbol{P}_{i}^{\prime}, V_{i}^{\prime}$; - see text for definitions).

\begin{tabular}{|c|c|c|c|c|}
\hline $\begin{array}{l}\text { Reactions } \\
\text { Amplitudes }\end{array}$ & $\begin{array}{c}0^{-}+p \rightarrow 0^{-}+\left(n, \Delta^{0}\right) \\
(\mathrm{NP} \text { exchange })\end{array}$ & $\begin{array}{c}m=0 \\
\text { (NP exchange) }\end{array}$ & $\begin{array}{c}0^{-}+p \rightarrow 1^{-}+\left(n, \Delta^{0}\right) \\
m=+1 \\
\text { (UP exchange) }\end{array}$ & $\begin{array}{c}m=-1 \\
\text { (UP exchange) }\end{array}$ \\
\hline$p_{1 / 2} \rightarrow n_{+1 / 2}$ & $\frac{1}{g}\left[(9-10 x) P_{1}^{\prime}-2 x P_{2}^{\prime}\right]$ & $\left.\frac{1}{9}\left[(9-10 x) V_{1}^{\prime}-2 x V_{2}^{\prime}\right)\right]$ & 0 & 0 \\
\hline$p_{1 / 2} \rightarrow n_{-1 / 2}$ & 0 & 0 & $\left(1-\frac{8}{9} x\right) V_{3}^{\prime}$ & $\left(1-\frac{8}{9} x\right) V_{5}^{\prime}$ \\
\hline$p-1 / 2 \rightarrow n_{+1 / 2}$ & .0 & 0 & - $\quad\left(1-\frac{8}{9} x\right) V_{4}^{\prime}$ & $\left.1-\frac{8}{9} x\right) V_{6}^{\prime}$ \\
\hline$p_{-1 / 2} \rightarrow n_{-1 / 2}$ & $\frac{1}{9}\left[(9-10 x) P_{2}^{\prime}-2 x P_{1}^{\prime}\right]$ & $\frac{1}{9}\left[(9-10 x) V_{2}^{\prime}-2 x V_{1}^{\prime}\right]$ & 0 & 0 \\
\hline$p_{1 / 2} \rightarrow \Delta_{+3 / 2}^{0}$ & 0 & 0 & $-\frac{2 \sqrt{x}}{3 \sqrt{3}} V_{4}^{\prime}$ & $-\frac{2 \sqrt{x}}{3 \sqrt{3}} V_{6}^{\prime}$ \\
\hline$p_{1 / 2} \rightarrow \Delta_{1+1 / 2}^{0}$ & $\frac{2 \sqrt{x}}{9}\left(P_{1}^{\prime}-P_{2}^{\prime}\right)$ & $\frac{2 \sqrt{x}}{9}\left(V_{1}^{\prime}-V_{2}^{\prime}\right)$ & 0 & 0 \\
\hline$p_{1 / 2} \rightarrow \Delta_{-1 / 2}^{0}$ & 0 & 0 & $\frac{2 \sqrt{x}}{9} V_{3}^{\prime}$ & $\frac{2 \sqrt{x}}{9} V_{5}^{\prime}$ \\
\hline$p_{1 / 2} \rightarrow \Delta_{-3 / 2}^{0}$ & 0 & 0 & 0 & 0 \\
\hline$p-1 / 2 \rightarrow \Delta_{+3 / 2}^{0}$ & 0 & 0 & 0 & 0 \\
\hline$p-1 / 2 \rightarrow \Delta_{+1 / 2}^{0}$ & 0 & 0 & $-\frac{2 \sqrt{x}}{9} V_{4}^{\prime}$ & $-\frac{2 \sqrt{x}}{9} V_{6}^{\prime}$ \\
\hline$p_{-1 / 2} \rightarrow \Delta_{-1 / 2}^{0}$ & $\frac{2 \sqrt{x}}{9}\left(P_{1}^{\prime}-P_{2}^{\prime}\right)$ & $\frac{2 \sqrt{x}}{9}\left(V_{1}^{\prime}-V_{2}^{\prime}\right)$ & 0 & 0 \\
\hline$p_{-1 / 2} \rightarrow \Delta_{-3 / 2}^{0}$ & 0 & 0 & $\frac{2 \sqrt{x}}{3 \sqrt{3}} V_{3}^{\prime}$ & $\frac{2 \sqrt{x}}{3 \sqrt{3}} V_{5}^{\prime}$ \\
\hline
\end{tabular}

will not go into the details of these "commonwith-SU(6)" relations. Let us just mention that in this class belong the many relations between (single and double) spin statistical tensors for weakly decaying particles and/or strongly decaying resonances produced in quasi-two-body reactions ${ }^{17}$; these relations have been presented and (successfully) tested by many authors (e.g., Ref. 18). Some of the relations common to the diquark model and to SU(6) have not been previously published and/or compared to experimental data. An example of such a "common" relation is the inequality governing charge exchange:

$$
\frac{\bar{\sigma}_{N}(n)}{\bar{\sigma}_{N}(\Delta)} \geqslant \frac{\bar{\sigma}_{U}(n)}{\bar{\sigma}_{U}(\Delta)},
$$

valid-by definition-for reactions with an $M=1^{-}$ meson. ${ }^{19}$

We now turn to the relations derivable from our model which are different from the corresponding SU(6) predictions.

(i) Predictions for strangeness-exchange reactions:

$$
\frac{3(1-x)^{2}}{x^{2}}[\bar{\sigma}(\Sigma)+2 x \bar{\sigma}(Y)]=\bar{\sigma}(\Lambda),
$$

where $x=\sin ^{2} \Gamma$ (as before). This relation replaces the $\mathrm{SU}(6)$ relation $^{20}$

$$
3[\bar{\sigma}(\Sigma)+\bar{\sigma}(Y)]=\bar{\sigma}(\Lambda)
$$

and is valid for the natural-parity (NP) exchange $\left(\bar{\sigma}_{N}\right)$ and unnatural-parity (UP) exchange $\left(\bar{\sigma}_{U}\right)$ cross sections separately. For UP exchange we find in addition

$$
\bar{\sigma}_{U}(\Lambda): \bar{\sigma}_{U}(\Sigma): \bar{\sigma}_{U}(Y)=27(1-x)^{2}: x^{2}: 4 x
$$

replacing the symmetric-quark-model prediction $^{3,7}$

$$
\bar{\sigma}_{U}(\Lambda): \bar{\sigma}_{U}(\Sigma): \bar{\sigma}_{U}(Y)=27: 1: 8 \text {. }
$$

The strong disagreement of relations (8) and (10) with the experimental data from $K^{-} p \rightarrow M^{0}$ $+(\Lambda, \Sigma, Y)$ was first discussed by Hirsch et $a l .^{3,7}$ These authors analyzed the problem in terms of baryon spin flip and spin nonflip. The baryonflip contributions obey relations identical to (9); the nonflip contributions obey relations equivalent to the sum rule (7).

For NP exchange we also have the relation

$$
P_{N}^{m}(\Lambda) \bar{\sigma}_{N}(\Lambda)=-9\left(\frac{1-x}{x}\right)^{2} P_{N}^{m}(\Sigma) \bar{\sigma}_{N}(\Sigma)
$$

instead of the $\mathrm{SU}(6)$ relation ${ }^{4}$ 


$$
P_{N}^{m}(\Lambda) \bar{\sigma}_{N}(\Lambda)=-9 P_{N}^{m}(\Sigma) \bar{\sigma}_{N}(\Sigma),
$$

where the $P^{m}$ is the polarization ${ }^{21}$ of the hyperon. $\left[P_{N}^{m}(Y)=0\right.$ is a prediction common to $\mathrm{SU}(6)$ and the diquark model.] As required, all out diquark-model predictions reduce to the corresponding $\mathrm{SU}(6)$ ones for $x=\frac{1}{2}(\Gamma=\pi / 4)$.

(ii) Predictions for charge-exchange reactions: For $M=1^{-}$we have that

$$
\bar{\sigma}_{U}(n)=\frac{(9-8 x)^{2}}{16 x} \bar{\sigma}_{U}(\Delta)
$$

and in general (both for $M=0^{-}$and $1^{-}$)

$$
\bar{\sigma}(n) \geqslant \frac{(9-8 x)^{2}}{16 x} \bar{\sigma}(\Delta)
$$

instead of the quark-SU(6)-model relation ${ }^{22}$

$$
\bar{\sigma}(n) \geqslant \frac{25}{8} \bar{\sigma}(\Delta) \text {. }
$$

Relation (14) is obtained by expressing the cross section ratio in terms of the quantity $x$ and the meson-quark transversity amplitudes $P_{i}^{\prime}$ and $V_{i}^{\prime}$ of Table IV and minimizing the resulting expression in these variables.

One final remark should be made: All relations derived here, although written down for total cross sections, are in principle only valid for the peripheral part of the cross section (both total and differential).

\section{COMPARISON WITH EXPERIMENT}

In order to check the relations derived in the preceding section it is usually necessary, because of the lack of data, to integrate over (frequently quite large) intervals in $t$. Sometimes we shall use total cross sections. Although our model can be expected to be valid only for peripheral interactions, the relative smallness of the backward production cross sections should not unduly influence these comparisons. Where we are able to use only peripheral cross sections, we will integrate over equal intervals of $t^{\prime}=\left|t-t_{\min }\right|$ instead of $t$ in order to reduce purely kinematical effects in the very forward direction.

Before being able to compare our model predictions with experiment, we must also adopt a prescription for treating the differences in kinematic factors resulting from mass differences. This is a standard difficulty when comparing symmetry predictions with experiment for which there is at present no rigorous solution. Let us first stress that all our comparisons will be performed between reactions with the same initial state (both in terms of beam momentum and particle type). Furthermore, although we will occasionally go as low in the beam momentum as $3 \mathrm{GeV} / c$, most of our comparisons will be at
$4 \mathrm{GeV} / c$ or higher; the latter implies a variation in $Q$ values (or $p^{*}$ c.m. momentum) between the different inelastic final states of at most $25 \%$ (or $15 \%$ ) respectively.

The prescription which is most often used in the literature is the one given by Meshkov et al. ${ }^{23}$ consisting of comparing cross sections $\bar{\sigma}(d \bar{\sigma} / d t)$ given by

$$
\bar{\sigma}=s \frac{p_{i}^{*}}{p_{f}^{*}} \sigma,
$$

where $\sqrt{s}$ is the total $c_{0}$ m. energy and $p_{i}^{*}, p_{f}^{*}$ are c.m. momenta for the initial and final state, respectively. In addition, according to Meshkov et al. such a comparison should be performed at equal $Q$-values.

Trilling ${ }^{24}$ has convincingly pointed out that the above prescription can lead to serious and unphysical discrepancies which are avoided if one compares reduced cross sections given by

$$
\bar{\sigma}=s \frac{p_{i}^{*\left(2 l_{i+1}\right)}}{p_{f}^{*\left(2 l_{f+1}\right)}} \sigma
$$

at equal $s$ values. In (17) $l_{i}$ and $l_{f}$ are the average (or dominating) orbital angular momentum in initial and final states. Apart from using data at equal $s$ instead of at equal $Q$, this prescription differs from that of Meshkov et al. in its inclusion of angular momentum barrier factors. One problem with this prescription is the introduction of at least one new parameter. With impact-parameter considerations one can derive limits for this parameter but in essence it remains an adjustable quantity. In general more parameters make model predictions less convincing. We will therefore as a rule use the Trilling prescription but without the angular barrier factor. This is equivalent to the prescription (16) but now at equal $s$ instead of equal $\boldsymbol{Q}$. Apart from the arguments given by Trilling we feel that the latter assumption is also not warranted by the $s$ regions considered here. Whenever relevant we will point out how our conclusions would be affected by using the complete Trilling prescription. Also, we have verified that our conclusions are qualitatively independent of whether one compares at equal $s$ or at equal $Q$. It will be argued that within the present quarkdiquark model all resulting differences are largely absorbed by relatively small differences in the breaking angle $\Gamma$.

Finally, we need to adopt a value of $x=\sin ^{2} \Gamma$. While making a fit to all available data might seem to be the best method of determining $x$, in practice such a fit poses severe problems not the least of which is the evaluation of the errors in a large number of data. We have therefore chosen to simply determine $x$ from the cross sections 
for $K^{-} p-\Lambda \rho$ and $K^{-} p \rightarrow Y \rho$ in our high-statistics $(\sim 130$ events $/ \mu \mathrm{b}) 4.2-\mathrm{GeV} / c K^{-} p$ experiment (see Table VII). A direct evaluation of Eq. (9) for these cross sections yields ${ }^{25} x=0.64 \pm 0.02$ or $\Gamma \approx 53^{\circ}$. All of our comparisons will be made using this value of $x$.

\section{A. Strangeness-exchange reactions}

For the strangeness-exchange reactions there are two types of processes which can be used to test our model, namely

$$
K^{-} p \rightarrow M+(\Lambda, \Sigma, Y) \text { with } M=\pi^{0}, \rho^{0}, \eta \text {, or } \phi
$$

and

$$
\pi^{-} p \rightarrow M+(\Lambda, \Sigma, Y) \text { with } M=K^{0}, K^{* 0} .
$$

Some of these reactions (e.g. $K^{-} p \rightarrow \pi^{0} \Sigma^{0}$ ) are quite difficult to measure; for such reactions we will use isospin-related reactions such as

$$
\sigma\left(K^{-} p \rightarrow \pi^{0} \Sigma^{0}\right)=\frac{1}{4} \sigma\left(K^{-} p \rightarrow \pi^{-} \Sigma^{+}\right) .
$$

For reactions producing an $I=0$ meson or $I=0$ baryon these relations follow directly from isospin conservation; otherwise the additional assumption of pure $I=\frac{1}{2}$ exchange must be made, an assumption already made in our model [and in the usual SU(6) quark model] where only single quark transitions are considered.

Tables $\mathrm{V}$ and VI show the tests of the diquark relation (7) vs the SU(6) relation (8) for $K^{-} p$ and $\pi \not p$ initial states, respectively. Table VII tests the diquark relation (9) vs the $S U(6)$ relation (10) for $K^{-} p$ reactions. The agreement between the data and the diquark-model predictions is surprisingly good while in most cases the $\mathrm{SU}(6)$ prediction is in strong disagreement with the data. We wish to point out that the use of the complete Trilling prescription would not lessen the preference of the data for the diquark model. The gen-

\begin{tabular}{|c|c|c|c|c|c|c|}
\hline $\begin{array}{c}\text { Reaction } \\
\left(M^{0}\right)\end{array}$ & $\bar{\sigma}(\Sigma)=\frac{p_{A}^{*}}{p_{\Sigma}^{*}} \sigma(\Sigma)$ & $\bar{\sigma}(Y)=\frac{p_{A}^{*}}{p_{Y}^{*}} \sigma(Y)$ & $\begin{array}{c}\text { LHS } \\
\text { SU(6) } \\
\text { relation }\end{array}$ & $\begin{array}{c}\text { LHS } \\
\text { diquark } \\
\text { relation }\end{array}$ & $\bar{\sigma}(\Lambda)=\sigma(\Lambda)$ & Ref. \\
\hline \multicolumn{7}{|c|}{$p_{\text {lab }}=3 \mathrm{GeV} / c$ (forward hemisphere) } \\
\hline$\pi^{0}$ & $108 \pm 18$ & $70 \pm 20$ & $534 \pm 81$ & $193 \pm 31$ & $233 \pm 30$ & 26 \\
\hline$\eta$ & $37 \pm 6$ & $59 \pm 12$ & $288 \pm 40$ & $106 \pm 16$ & $100 \pm 26$ & 26 \\
\hline$\rho^{0}$ & $85 \pm 7$ & $75 \pm 10$ & $480 \pm 37$ & $177 \pm 14$ & $219 \pm 50$ & 26 \\
\hline$\rho^{0}$ & $91 \pm 13$ & $84 \pm 14$ & $525 \pm 57$ & $193 \pm 22$ & $160 \pm 54$ & 3,27 \\
\hline$\omega$ & $94 \pm 12$ & $84 \pm 23$ & $534 \pm 78$ & $197 \pm 31$ & $196 \pm 36$ & 3,27 \\
\hline$\phi$ & $51 \pm 10$ & $59 \pm 15$ & $330 \pm 54$ & $123 \pm 21$ & $82 \pm 20$ & 26 \\
\hline \multicolumn{7}{|c|}{$p_{\text {lab }}=3.9 \mathrm{GeV} / c$ (Forward hemisphere) } \\
\hline$\rho^{0}$ & $26 \pm 5$ & $26 \pm 2$ & $157 \pm 17$ & $57 \pm 6$ & $63 \pm 9$ & 28 \\
\hline$\phi$ & $40 \pm 8$ & $21 \pm 5$ & $183 \pm 30$ & $63 \pm 10$ & $59 \pm 7$ & 28 \\
\hline \multicolumn{7}{|c|}{$p_{\text {lab }}=4.6 \mathrm{GeV} / c$ (forward hemisphere) } \\
\hline$\rho^{0}$ & $20 \pm 3$ & $17 \pm 3$ & $110 \pm 13$ & $40 \pm 4$ & $45 \pm 5$ & 28 \\
\hline$\phi$ & $23 \pm 6$ & $19 \pm 4$ & $125 \pm 21$ & $44 \pm 7$ & $41 \pm 4$ & 28 \\
\hline \multicolumn{7}{|c|}{$p_{\mathrm{lab}}=14.3 \mathrm{GeV} / c\left(t^{\prime}<1.0 \mathrm{GeV}^{2}\right)$} \\
\hline$\pi^{0}$ & $5.1 \pm 0.5$ & $2.1 \pm 0.2$ & $22 \pm 2$ & $7.6 \pm 0.5$ & $8.4 \pm 1.2$ & 29,3 \\
\hline \multicolumn{7}{|c|}{$p_{\mathrm{lab}}=4.2 \mathrm{GeV} / c\left(t^{\prime}<1.0\right.$ or $\left.1.2 \mathrm{GeV}^{2}\right)$} \\
\hline$\pi^{0 \mathrm{a}}$ & $31 \pm 2$ & $20 \pm 1$ & $153 \pm 7$ & $55 \pm 2$ & $61 \pm 3$ & 30 \\
\hline $\begin{array}{l}\rho^{0} \mathrm{~b} \\
\rho^{0} \mathrm{NP}\end{array}$ & $17 \pm 2$ & $20 \pm 3$ & $111 \pm 11$ & $42 \pm 2$ & $46 \pm 3$ & 6,31 \\
\hline $\begin{array}{l}\text { exchange } \\
\rho^{0} \text { UP }\end{array}$ & $14 \pm 1$ & $4 \pm 1$ & $54 \pm 4$ & $19 \pm 2$ & $24 \pm 2$ & 6,31 \\
\hline exchange & $3 \pm 1$ & $16 \pm 2$ & $57 \pm 7$ & $23 \pm 3$ & $22 \pm 2$ & 6,31 \\
\hline$\phi^{\mathrm{a}}$ & $28 \pm 2$ & $26 \pm 2$ & $162 \pm 8$ & $60 \pm 3$ & $58 \pm 3$ & 6,31 \\
\hline $\begin{array}{l}\phi \text { NP } \\
\text { exchange } \\
\phi \text { UP }\end{array}$ & $23 \pm 2$ & $8 \pm 1$ & $93 \pm 7$ & $32 \pm 2$ & $27 \pm 2$ & 6,31 \\
\hline exchange & $4 \pm 1$ & $18 \pm 2$ & $66 \pm 7$ & $26 \pm 3$ & $32 \pm 2$ & 6,31 \\
\hline
\end{tabular}

TABLE V. Test of diquark relation (7), $\left[3(1-x)^{2} / x^{2}\right][\bar{\sigma}(\Sigma)+2 x \bar{\sigma}(Y)]=\bar{\sigma}(\Lambda)$, versus SU (6) relation $(8), 3[\bar{\sigma}(\Sigma)+\bar{\sigma}(Y)]$ $=\bar{\sigma}(\Lambda)$ for $K^{-} P \rightarrow M^{0}+(\Lambda, \Sigma, Y)$ and $x \simeq 0.64$ (in $\mu \mathrm{b}$ units).

${ }^{a} t^{\prime}<1 \mathrm{GeV}^{2}$.

${ }^{\mathrm{b}} t^{\prime}<1.2 \mathrm{GeV}^{2}$. 
TABLE VI. Test of diquark relation (7) versus SU (6) relation (8) for $\pi^{-} p \rightarrow K^{0}\left(K^{* 0}\right)$ $+(\Lambda, \Sigma, Y)$ and $x \simeq 0.64$ (in $\mu \mathrm{b}$ units).

\begin{tabular}{|c|c|c|c|c|c|c|}
\hline Reaction & $\bar{\sigma}(\Sigma)=\frac{p_{A}^{*}}{p_{\Sigma}^{*}} \sigma(\Sigma)$ & $\tilde{\sigma}(Y)=\frac{p_{*}^{*}}{p_{Y}^{*}} \sigma(Y)$ & $\begin{array}{c}\text { LHS } \\
\text { SU(6) } \\
\text { relation }\end{array}$ & $\begin{array}{c}\text { LHS } \\
\text { diquark } \\
\text { relation }\end{array}$ & $\bar{\sigma}(\Lambda)=\sigma(\Lambda)$ & Ref. \\
\hline \multicolumn{7}{|c|}{$p_{\text {lab }}=3.9 \mathrm{GeV} / c \quad\left(t^{\prime}<1.0 \mathrm{GeV}^{2}\right)$} \\
\hline$K * 0$ & $32 \pm 3$ & $24 \pm 3$ & $168 \pm 13$ & $61 \pm 5$ & $57 \pm 4$ & 32 \\
\hline \multicolumn{7}{|c|}{$p_{\mathrm{lab}}=4.5 \mathrm{GeV} / c\left(t^{\prime}<1.0 \mathrm{GeV}^{2}\right)$} \\
\hline$K^{0}$ & $27 \pm 3$ & $14 \pm 3$ & $123 \pm 13$ & $43 \pm 5$ & $50 \pm 5$ & 33 \\
\hline
\end{tabular}

eral effect is a (relative) increase of the LHS of relation (7) and (8); however, this just results in a new value for $x$ (e.g., $0.66 \pm 0.02$ for $l_{f}=1$ ) which largely compensates the increase. The net result is that the $\mathrm{SU}(6)$ relations become worse while the diquark relations remain well satisfied. As an example, for $\phi$ production (see Table VII), the $\bar{\sigma} f$ values become $\bar{\sigma}(\Sigma) f_{\mathrm{dq}}=(37 \pm 9) \mu \mathrm{b}, \bar{\sigma}(\Sigma) f_{\mathrm{SU}(6)}$ $=(138 \pm 35) \mu \mathrm{b}, \bar{\sigma}(Y) f_{\mathrm{dq}}=(30 \pm 4) \mu \mathrm{b}$, and $\bar{\sigma}(Y) f_{\left.\mathrm{SU}_{6}\right)}$ $=(84 \pm 10) \mu \mathrm{b}$. All these values must still be compared with $\bar{\sigma}(\Lambda)=(32 \pm 2) \mu \mathrm{b}$.

Thus far we have examined cross sections integrated over large $t^{\prime}$ intervals. We now turn to an examination of differential cross sections using the high statistics of our $4.2-\mathrm{GeV} / c \mathrm{~K}^{-} \mathrm{p}$ experiment and concentrating on natural-parity-exchange reactions $\left(M=\pi^{0}\right)$ or on the natural-parity-exchange part of reactions $\left(M=\rho^{0}, \phi^{0}\right)$. The method consists of combining the relations (7) and (8) with (11) and (12), respectively, by performing amplitude fits in each $t^{\prime}$ interval separately in a manner analogous to what was done in Ref. 4. As may be seen in Table III, for each choice of meson there are only two independent naturalparity-exchange meson-quark amplitudes. In each $t^{\prime}$ bin we can then fit the two amplitude magnitudes and one relative phase to five quantities $\left(d \sigma_{N} / d t^{\prime}\right.$ for $\Lambda, \Sigma, Y$, and $P_{N}^{m}$ for $\left.\Lambda, \Sigma\right)$. For $M=\pi^{0}$ (Ref. 30 ) we show the fitted results in Fig. 2. Solid (broken) lines connect the results of the diquark-model [SU(6)-model] fits. There is little difference between the polarization fit results of the two models (only the diquark result is shown), which is not surprising since the polarizations are independent of $x$. Once more we observe that the diquark model is in reasonable agreement with the data while $\mathrm{SU}(6)$ is again in violent disagreement. Also shown are the fitted values of the two amplitudes and their relative phase from the diquark fits. The relatively smooth variation of these quantities as a function of $t^{\prime}$ increases confidence in the fits. We also note that the amplitudes are consistent with being equal in magnitude and having relative phase $=0$ at $t^{\prime}=0$; this is required (for the production of a spin-0 meson) by parity and angular momentum conservation if one assumes $J=0$ or $J=1$ exchange only [as both the diquark and SU(6) models do]. For the same reasons $d \bar{\sigma} / d t^{\prime}$ must be zero at $t^{\prime}=0$ for $K^{-} p \rightarrow \pi^{0} Y$ (Ref. 34). Relations (7) and (8) then predict that the ratios of the $d \bar{\sigma} / d t^{\prime}$ for $K^{-} p \rightarrow \pi^{0} \Lambda$ and $K^{-} p \rightarrow \pi^{0} \Sigma^{-}$at $t^{\prime}=0$ must be 0.98 for the diquark model and 3.0 for $\mathrm{SU}(6)$. Our data are again reasonably compatible with the diquark model and in strong disagreement with the SU(6) predictions. This ratio has also been measured with great precision at $8,10.7$, and $15.7 \mathrm{GeV} / c$ to be $1.10 \pm 0.10,1.15 \pm 0.10$, and $1.10 \pm 0.15$, respectively. ${ }^{35}$ Again only the diquark-model predictions are confirmed.

We have performed similar fits and obtained similar results for the natural-parity-exchange parts of the reactions with $M=\rho^{0}$ and $M=\phi$, the only difference being that the statistics in these

TABLE VII. Test of diquark relation $(9), \bar{\sigma}_{U}(\Lambda): \bar{\sigma}_{U}(\Sigma): \bar{\sigma}_{U}(Y)=27(1-x)^{2}: x^{2}: 4 x$, versus $\mathrm{SU}(6)$ relation $(10), \bar{\sigma}_{U}(\Lambda): \bar{\sigma}_{U}(\Sigma): \bar{\sigma}_{U}(Y)=27: 1: 8$, for $K^{-} p \rightarrow \rho^{0}\left(\phi^{0}\right)+(\Lambda, \Sigma, Y)$ and $x \simeq 0.64$ (in $\mu \mathrm{b}$ units); $p_{\text {lab }}=4.2 \mathrm{GeV} / c . f_{\mathrm{dq}}=8.8$ and 8.8/6.3 for $\Sigma$ and $Y$, respectively; $f_{\text {SU(6) }}=27$ and $\frac{27}{8}$ for $\Sigma$ and $Y$, respectively.

\begin{tabular}{lccrccc}
\hline \hline Reaction & $\bar{\sigma}(\Lambda)$ & $\bar{\sigma}(\Sigma) f_{\mathrm{dq}:}$ & $\bar{\sigma}(\Sigma) f_{\mathrm{SU}(6)}$ & $\bar{\sigma}(Y) f_{\mathrm{dq}}$ & $\bar{\sigma}(Y) f_{\mathrm{SU}(6)}$ & Ref. \\
\hline$\rho^{0 \mathrm{a}}$ & $22 \pm 2$ & $26 \pm 9$ & $81 \pm 27$ & $22 \pm 3$ & $54 \pm 7$ & 6,31 \\
$\phi^{\mathrm{b}}$ & $32 \pm 2$ & $35 \pm 9$ & $108 \pm 27$ & $25 \pm 3$ & $61 \pm 7$ & 6,31 \\
\hline \hline
\end{tabular}

${ }^{\mathrm{a}} \boldsymbol{t}^{\prime}<1.2 \mathrm{GeV}^{2}$.

$\mathrm{b}_{\boldsymbol{t}^{\prime}}<1.0 \mathrm{GeV}^{2}$. 

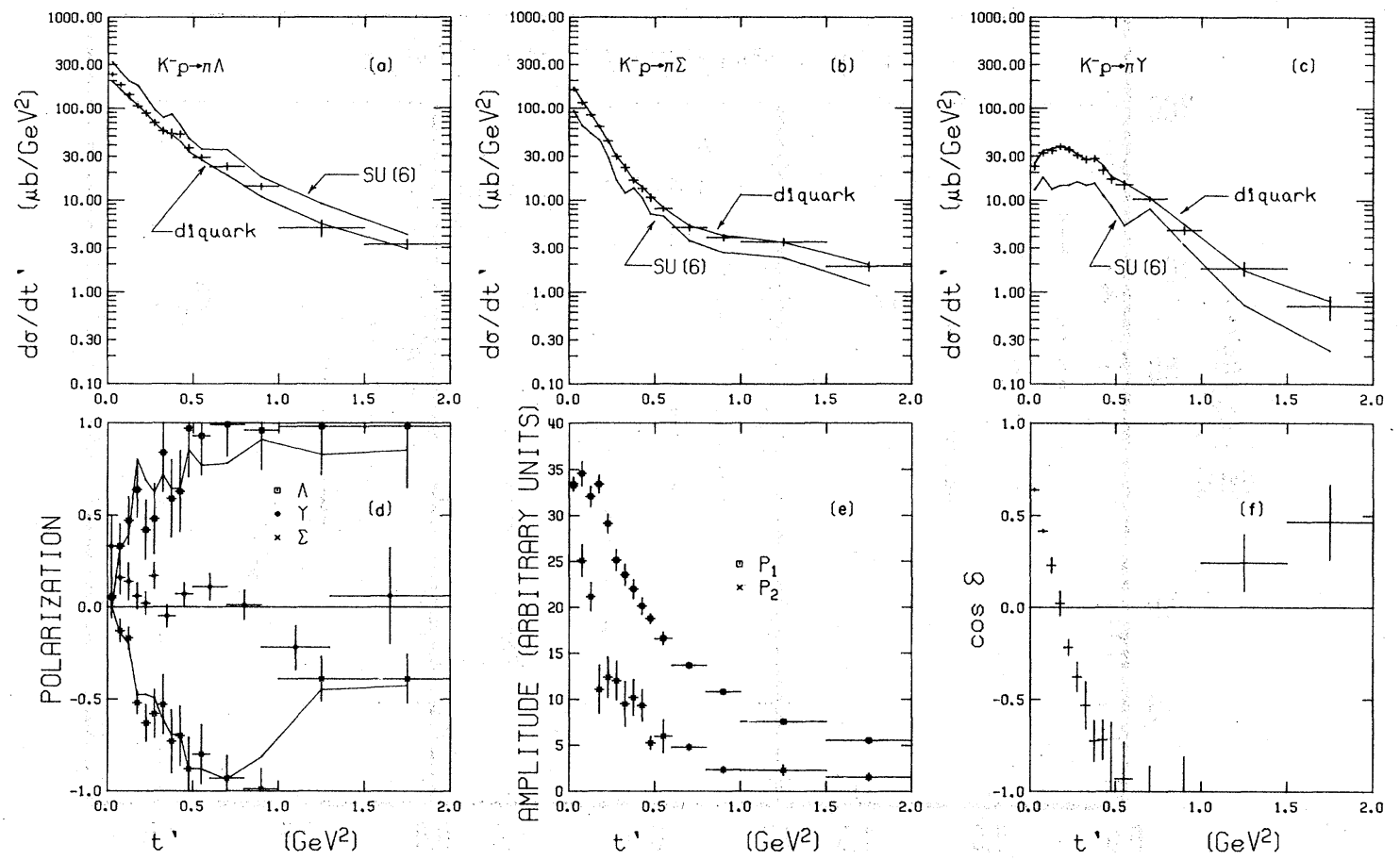

FIG. 2. (a)-(d) Differential cross sections and polarization for $K^{-} p \rightarrow \pi^{0}(\Lambda, \Sigma, Y)$ at $4.2 \mathrm{GeV} / c$ compared with the results of fits to the diquark and SU(6) quark transversity amplitudes as described in the text; (e) the magnitudes of these diquark transversity amplitudes and (f) their relative phase as found in the fits.

channels is lower, and the results therefore are somewhat less significant. For these reactions we show only a comparison of the differential cross sections..$^{6.31}$ Figures $3(a)$ and $3(b)$ show the comparison (for different $t^{\prime}$ values) of the left-hand side of relations (7) and (8) with their corresponding right-hand side for $\bar{\sigma}, \bar{\sigma}_{N}$, and $\bar{\sigma}_{U}$. While the SU(6) quark model disagrees violently with the data, the diquark model is in good agreement in nearly all cases; only the natural-exchange part of the $\rho^{0}$ reaction shows discrepancies of a few standard deviations at intermediate $t^{\prime}$ values, but even here the diquark model is in much better agreement with the data than SU(6).

One final remark: Assuming vector dominance, photoproduction of strange baryons should also obey relation (7) or (8). Experimentally one finds $^{36}$ ( $k=$ incident photon energy)

$$
\sigma\left(\gamma p \rightarrow K^{+} \Lambda\right)=(6.7 \pm 0.04) k^{-2} \mu \mathrm{b}
$$

to be compared with the left-hand side of $(6.7 \pm 1.2) k^{-2} \mu \mathrm{b}$ for the diquark model and $(19.8 \pm 31) k^{-2} \mu \mathrm{b}$ for the SU(6) model. Once more the diquark model agrees; the SU(6) model does not.

\section{B. Charge-exchange reactions}

For the charge-exchange reactions there are three types of reactions which allow a test of re- lations (13), (14), and (15) namely:

$$
\begin{aligned}
& K^{-} p \rightarrow M^{0}+(n, \Delta) \text { with } M^{0}=\bar{K}^{0}, \bar{K}^{* 0}, \\
& K p \rightarrow M^{+}+(n, \Delta) \text { with } M^{+}=K^{+}, K^{*+}, \\
& \pi^{-} p \rightarrow M^{0}+(n, \Delta) \text { with } M^{0}=\pi^{0}, \rho^{0}, \omega, \text { or } \phi .
\end{aligned}
$$

Here too we will use isospin relations to avoid experimentally difficult reactions, e.g.

$$
\sigma\left(\pi^{-} p \rightarrow \pi^{0} \Delta^{0}\right)=\frac{1}{3} \sigma\left(\pi^{+} p \rightarrow \pi^{0} \Delta^{++}\right)
$$

and

$$
\sigma\left(K^{0} p \rightarrow K^{+} \Delta^{0}\right)=\frac{1}{3} \sigma\left(K^{+} p \rightarrow K^{0} \Delta^{++}\right) .
$$

Most of these relations result from pure isospin conservation. Only those with $I=1$ mesons and a $\Delta$ in the final state [reactions (22)] require the assumption of no $I=2$ exchange in the $t$ channel (or equivalently only single-quark transitions).

First we make a test of the equality (13) using our $4.2-\mathrm{GeV} / c K^{*} p \rightarrow K^{* 0}(n, \Delta)$ data..$^{37,38}$ The diquark-model prediction for $\bar{\sigma}_{U}(n) / \bar{\sigma}_{U}(\Delta)$ is 1.5; the $S U(6)$ value is 3.1. Experimentally we find $1.3 \pm 0.4$. The diquark model is strongly favored. Here again we find that using the complete Trilling prescription increases the preference for the diquark model: The diquark prediction shifts to 1.3 , again as a result of a slightly increased $x$; that of $\mathrm{SU}(6)$ remains 3.1 (by definition) while the experimental value becomes $1.0 \pm 0.3$. 

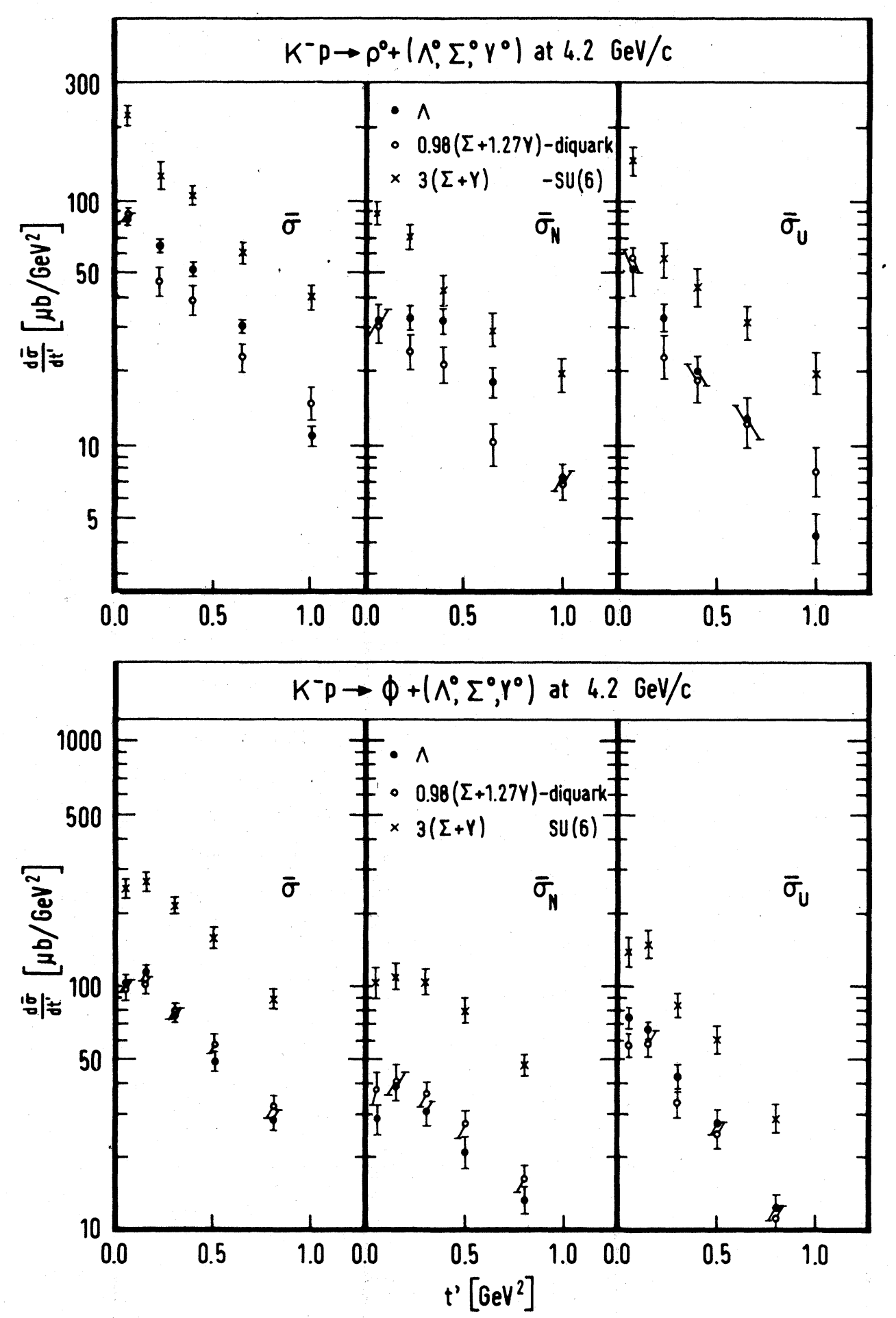

FIG. 3. Comparison of the left-hand sides of relations (7) and (8) with their right-hand side.

In Tables VIII and IX we present a test of relations (14) and (15) for $K^{-} p$ and $K^{0} p$ initial states, respectively. The agreement with the diquark model is remarkable; only one entry is incon- clusive (although compatible). Examination of the forward differential cross sections for the $K^{-} p$ reactions at $5,8.4,12.8,13$, and $15.7 \mathrm{GeV} / c$ shows that this conclusion is also valid at each $t$. 
TABLE VIII. Test of diquark relation (14), $\bar{\sigma}(n) \geq(9-8 x)^{2} / 16 x \bar{\sigma}(\Delta)$, versus the SU(6) relation (15), $\bar{\sigma}(n) \geq \frac{25}{8} \bar{\sigma}(\Delta)$, for $K^{-} p \rightarrow \bar{K}^{0}(\bar{K} * 0)+(n, \Delta)$ and $x \simeq 0.64$ (in $\mu \mathrm{b}$ units). $f_{\mathrm{dq}} \simeq 1.50, f_{\mathrm{SU}(6)}=\frac{25}{8}$.

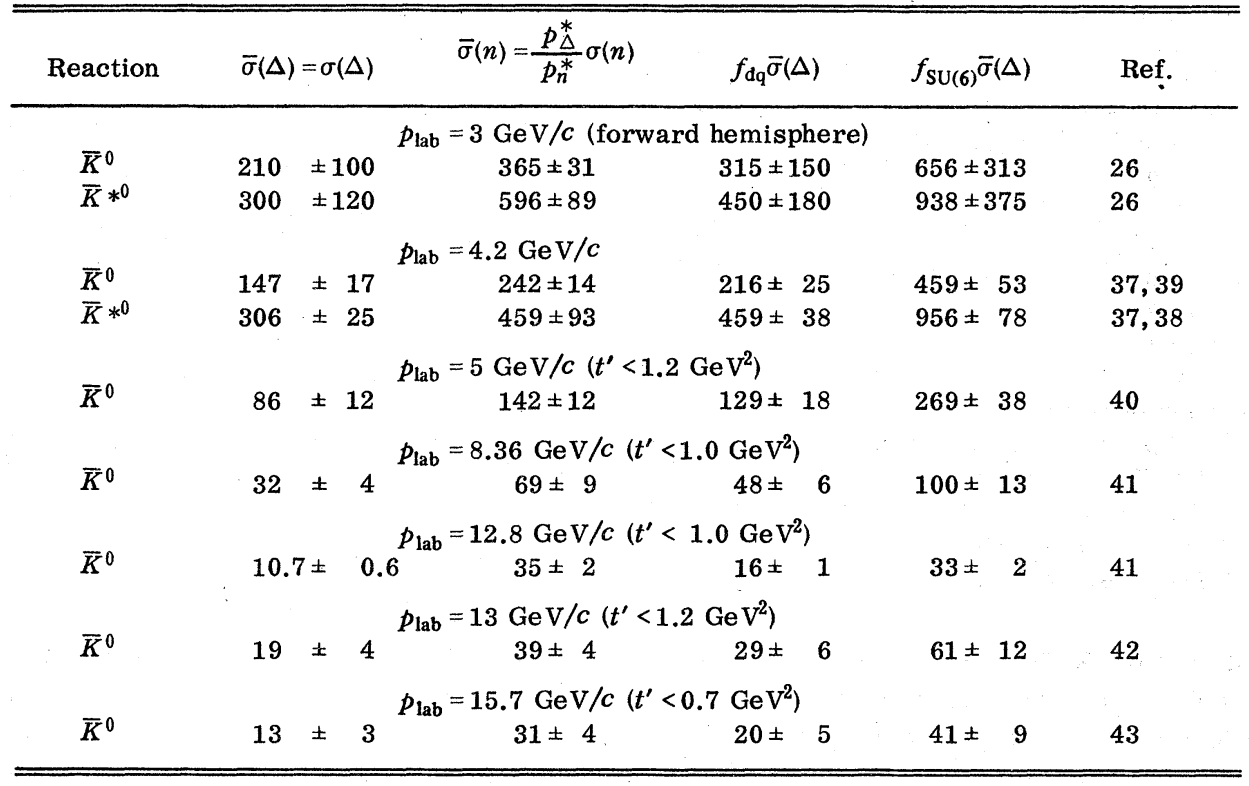

For the pion-induced reactions we are able to test directly the equality (13) for the unnaturalparity exchange using the reactions $\pi^{+} n \rightarrow \omega p$ at 6.95 GeV/c (Ref. 49) and $\pi^{+} p \rightarrow \omega \Delta^{+4}$ at $7.1 \mathrm{GeV} / c$ (Ref. 50) which are related to reactions (22) by isospin. We have extracted the UP cross section for $0.06<t<0.50 \mathrm{GeV}^{2}$ and obtained

$$
\bar{\sigma}_{U}(n)=\frac{p_{\Delta}^{*}}{p_{n}^{*}} \sigma_{U}(n)=(9.8 \pm 2.5) \mu \mathrm{b}
$$

$$
\bar{\sigma}_{U}(\Delta)=\sigma_{U}(\Delta)=(7.4 \pm 0.5) \mu \mathrm{b} .
$$

The equality (13) is then

$$
(9.8 \pm 2.5) \mu \mathrm{b}=(11.1 \pm 0.8) \mu \mathrm{b} \text { (diquark) }
$$

or

$$
(9.8 \pm 2.5) \mu \mathrm{b}=(23.1 \pm 1.6) \mu \mathrm{b}[\mathrm{SU}(6)] .
$$

Again, the diquark model agrees well with the data

\begin{tabular}{|c|c|c|c|c|c|}
\hline Reaction & $\bar{\sigma}(\Delta)=\sigma(\Delta)^{\mathrm{a}}$ & $\vec{\sigma}(n)=\frac{p_{\Delta}^{*}}{p_{n}^{*}} \sigma(n)^{\mathrm{b}}$ & $f_{\mathrm{dq}} \bar{\sigma}(\Delta)$ & $f_{\mathrm{SU}(6)} \bar{\sigma}(\Delta)$ & Ref. \\
\hline & & $p_{\mathrm{lab}}=2.3 \mathrm{GeV} / c$ & & & \\
\hline \multirow[t]{2}{*}{$K^{+}$} & $530 \pm 33$ & $1222 \pm 131$ & $795 \pm 50$ & $1656 \pm 103$ & 44 \\
\hline & & $p_{\text {lab }}=2.97 \mathrm{GeV} / c$ & & & \\
\hline$K^{+}$ & $630 \pm 90$ & $653 \pm 70$ & $945 \pm 135$ & $1970 \pm 280$ & 45,46 \\
\hline \multirow[t]{2}{*}{$K^{*+}$} & $337 \pm 67$ & $1019 \pm 102$ & $506 \pm 100$ & $1053 \pm 209$ & 45,47 \\
\hline & & $p_{\text {lab }}=8.36 \mathrm{GeV} / c$ & $\left(t^{\prime}<1.0 \mathrm{GeV}^{2}\right)$ & & \\
\hline \multirow[t]{2}{*}{$K^{+}$} & $34 \pm 5$ & $94 \pm 13$ & $51 \pm \quad 8$ & $106 \pm 17$ & 41 \\
\hline & & $p_{\mathrm{lab}}:=10 \mathrm{GeV} / c$ & & & \\
\hline \multirow[t]{2}{*}{$K^{+}$} & $24 \pm 3$ & $74 \pm 13$ & $36 \pm \quad 5$ & $75 \pm 10$ & 48 \\
\hline & & $p_{\mathrm{lab}} \pm 12.8 \mathrm{GeV} / c$ & $\left(t^{\prime}<1.0 \mathrm{GeV}^{2}\right)$ & & \\
\hline$K^{+}$ & $17 \pm 2$ & $49 \pm \quad 6$ & $25 \pm 3$ & $52 \pm 6$ & 41 \\
\hline
\end{tabular}
while $\mathrm{SU}(6)$ is in complete disagreement.

TABLE IX. Test of diquark relation (14) versus SU(6) relation (15) for $K^{0} p \rightarrow K^{+}\left(K^{*+}\right)$ $+(n, \Delta)$ and $x \simeq 0.64$ (in $\mu \mathrm{b}$ units). $f_{\mathrm{dq}} \approx 1.50, f_{\mathrm{SU}(6)}=\frac{25}{8}$.

${ }^{\text {a }}$ Derived from isospin relation $\sigma\left(K^{0} p \rightarrow K^{+}\left(K^{*^{+}}\right) \Delta^{0}\right)=\frac{1}{3} \sigma\left(K^{+} p \rightarrow K^{0}\left(K^{*}\right) \Delta^{++}\right)$.

${ }^{\mathrm{b}}$ Derived from isospin relation $\sigma\left(K^{0} p \rightarrow K^{+}\left(K^{*+}\right) n\right)=\sigma\left(K^{+} n \rightarrow K^{0}\left(K^{* 0}\right) p\right)$. 
TABLE X. Test of diquark relation (14) versus $\mathrm{SU}(6)$ relation (15) for $\pi^{-} p \rightarrow \pi^{0}+(n, \Delta)$ and $x \simeq 0.64$ (in $\mu \mathrm{b}$ units). $f_{\mathrm{dq}} \simeq 1.50, f_{\mathrm{SU}(6)}=\frac{25}{8}$.

\begin{tabular}{cccccc}
\hline $\begin{array}{c}p_{\text {lab }} \\
(\mathrm{GeV} / c)\end{array}$ & $\bar{\sigma}(\Delta)=\sigma(\Delta)^{\mathrm{a}}$ & $\bar{\sigma}(n)={\frac{p_{\Delta}^{*}}{p_{n}^{*}} \sigma(n)^{\mathrm{b}}}^{\mathrm{c}}$ & $f_{\mathrm{dq}} \bar{\sigma}(\Delta)$ & $f_{\mathrm{SU}(6)} \bar{\sigma}(\Delta)$ & Ref. \\
\hline 3.9 & $130 \pm 7^{\mathrm{c}}$ & $160 \pm 15$ & $195 \pm 11$ & $406 \pm 22$ & 52 \\
4.0 & $117 \pm 23$ & $154 \pm 15$ & $176 \pm 35$ & $366 \pm 72$ & 53 \\
5.0 & $73 \pm 3$ & $113 \pm 11$ & $109 \pm 5$ & $228 \pm 10$ & 54 \\
5.45 & $56 \pm 10$ & $102 \pm 8$ & $85 \pm 15$ & $175 \pm 31$ & 55 \\
8.0 & $37 \pm 3$ & $63 \pm 5$ & $56 \pm 5$ & $116 \pm 9$ & 56 \\
11.7 & $25 \pm 3^{\mathrm{d}}$ & $41 \pm 3$ & $38 \pm 5$ & $156 \pm 21$ & 57 \\
13.1 & $15 \pm 2$ & $36 \pm 2$ & $22 \pm 4$ & $47 \pm 7$ & 58 \\
16.0 & $18 \pm 1^{\mathrm{e}}$ & $29 \pm 2$ & $28 \pm 1$ & $58 \pm 2$ & 59 \\
\hline
\end{tabular}

${ }^{\mathrm{a}} \sigma\left(\pi^{-} p \rightarrow \pi^{0} \Delta^{0}\right)$ derived from isospin relation $\sigma\left(\pi^{-} p \rightarrow \pi^{0} \Delta^{0}\right)=\frac{1}{3} \sigma\left(\pi^{+} p \rightarrow \pi^{0} \Delta^{++}\right)$.

${ }^{\mathrm{b}}$ All $\pi^{-} p \rightarrow \pi^{0} n$ cross sections derived from the data of Ref. 51 for $t<1.5 \mathrm{GeV}^{2}$.

${ }^{c}$ Forward hemisphere.

d Corrected for $t<1.5 \mathrm{GeV}^{2}$.

e The cross section for $t^{\prime}>1.0 \mathrm{GeV}^{2}$ is consistent with zero.

In Tables $\mathrm{X}$ through XIII we again test relations (14) and (15) but now for various pion-induced processes. Table $X\left(\pi^{0}\right)$ and, in particular, Table XI $\left(\rho^{0}\right)$ refer to reactions whose cross sections are difficult to measure; they certainly contain nondetected systematic errors not accounted for in the quoted errors. (See e.g. the cross sections for $\rho^{0} \Delta^{0}$ at 13.0 and $13.1 \mathrm{GeV} / c$ and the two values at $16.0 \mathrm{GeV} / c_{\text {.) }}$ Tables XII and XIII involve reactions with narrow resonances and their cross sections are thus easier to determine than e.g. the $\rho^{0}$ reactions. Examination of these tables indicates strong disagreement with $\mathrm{SU}(6)$ in all cases and very good agreement with the diquark model for the narrow resonances. Only in the $\rho^{0}$ case do we find a few points lying two or more standard deviations away from the diquark-model prediction. However, for all these points the disagreement with $\mathrm{SU}(6)$ is at least an order of magnitude larger. In view of the above arguments we consider the diquark relations remarkably well satisfied also for pion-induced reactions.

We note that the experimental value for $\bar{\sigma}(n) / \bar{\sigma}(\Delta)$ is in many cases close to the minimal value. From relations (13) and (14) we see that within the framework of the diquark model this implies that

$$
\frac{\bar{\sigma}_{N}(n)}{\bar{\sigma}_{U}(n)} \simeq \frac{\bar{\sigma}_{N}(\Delta)}{\bar{\sigma}_{U}(\Delta)}
$$

Such a minimal value of $\bar{\sigma}(n) / \bar{\sigma}(\Delta)$ also implies that the baryon transition is entirely spin flip. Our 4.2-GeV $/ c$ data for the reaction $K^{-} p \rightarrow K^{0}+(n, \Delta)$

TABLE XI. Test of diquark relation (14) versus SU(6) relation (15) for $\pi^{-} p \rightarrow \rho^{0}+(n, \Delta)$ and $x \simeq 0.64$ (in $\mu \mathrm{b}$ units). $f_{\mathrm{dq}} \simeq 1.50, f_{\mathrm{SU}(6)}=\frac{25}{8}$.

\begin{tabular}{cccrrr}
\hline $\begin{array}{c}p_{\text {lab }} \\
(\mathrm{GeV} / c)\end{array}$ & $\bar{\sigma}(\Delta)=\sigma(\Delta)^{\mathrm{a}}$ & $\bar{\sigma}(n)=\frac{p_{\Delta}^{*}}{p_{n}^{*}} \sigma(n)^{\mathrm{b}}$ & $f_{\mathrm{dq}} \bar{\sigma}(\Delta)$ & $f_{\mathrm{SU}(6)} \bar{\sigma}(\Delta)$ & Ref. \\
\hline 3.9 & $500 \pm 13$ & $921 \pm 27^{\mathrm{c}}$ & $750 \pm 20$ & $1563 \pm 41$ & 52,60 \\
4.08 & $373 \pm 30$ & $917 \pm 36$ & $560 \pm 45$ & $1166 \pm 94$ & 62 \\
5.0 & $290 \pm 27$ & $604 \pm 37$ & $435 \pm 41$ & $906 \pm 84$ & 63 \\
5.0 & $343 \pm 33$ & $604 \pm 37$ & $515 \pm 50$ & $1072 \pm 103$ & 64 \\
5.45 & $238 \pm 14$ & $505 \pm 37$ & $357 \pm 21$ & $744 \pm 44$ & 65 \\
7.1 & $204 \pm 8$ & $288 \pm 19$ & $306 \pm 12$ & $638 \pm 25$ & 50 \\
8.0 & $104 \pm 12$ & $220 \pm 19$ & $156 \pm 18$ & $325 \pm 38$ & 56 \\
11.7 & $107 \pm 13$ & $97 \pm 15$ & $161 \pm 20$ & $334 \pm 41$ & 66 \\
13.0 & $57 \pm 7$ & $76 \pm 15$ & $86 \pm 11$ & $187 \pm 22$ & 67 \\
13.1 & $83 \pm 4$ & $75 \pm 15$ & $125 \pm 6$ & $259 \pm 13$ & 68 \\
16.0 & $41 \pm 7$ & $49 \pm 10$ & $62 \pm 11$ & $129 \pm 22$ & 69 \\
16.0 & $65 \pm 5$ & $49 \pm 10$ & $97 \pm 8$ & $202 \pm 17$ & 70 \\
18.5 & $29 \pm 7$ & $36 \pm 13$ & $44 \pm 11$ & $91 \pm 22$ & 71. \\
\hline \hline
\end{tabular}

${ }^{\mathrm{a}} \sigma\left(\pi^{-} p \rightarrow \rho^{0} \Delta^{0}\right)$ derived from the isospin relation $\sigma\left(\pi^{-} p \rightarrow \rho^{0} \Delta^{0}\right)=\frac{1}{3}\left(\pi^{+} p \rightarrow \rho^{0} \Delta^{++}\right)$.

${ }^{b} \pi^{-} p \rightarrow \rho^{0} n$ cross sections, with the exception of the $3.9-\mathrm{GeV} / c$ data derived from the data of Refs. 60 and 61.

${ }^{\mathrm{c}}$ Forward hemisphere. 
TABLE XII. Test of diquark relation (14) versus $S U(6)$ relation (15) for $\pi^{-} p \rightarrow \omega+(n, \Delta)$ and $x \simeq 0.64$ (in $\mu$ b units). $f_{\mathrm{dq}} \simeq 1.50, f_{\mathrm{SU}(6)}=\frac{25}{8}$.

\begin{tabular}{lrrrrr}
\hline $\begin{array}{c}p_{\text {iab }} \\
(\mathrm{GeV} / c)\end{array}$ & $\bar{\sigma}(\Delta)=\sigma(\Delta)^{\mathrm{a}}$ & $\bar{\sigma}(n)=\frac{p_{\Delta}^{*}}{p_{n}^{*}} \sigma(n)^{\mathrm{b}}$ & $f_{\mathrm{dq}} \bar{\sigma}(\Delta)$ & $f_{\mathrm{SU}(6)} \bar{\sigma}(\Delta)$ & Ref. \\
\hline 4.0 & $167 \pm 50$ & $276 \pm 22$ & $250 \pm 75$ & $522 \pm 156$ & 53 \\
4.09 & $120 \pm 10$ & $262 \pm 21$ & $180 \pm 15$ & $375 \pm 31$ & 73 \\
5.0 & $93 \pm 3$ & $164 \pm 18$ & $140 \pm 5$ & $291 \pm 9$ & 54,63 \\
5.45 & $98 \pm 6$ & $135 \pm 18$ & $147 \pm 9$ & $306 \pm 19$ & 65 \\
7.1 & $47 \pm 1$ & $71 \pm 17$ & $71 \pm 2$ & $147 \pm 6$ & 50 \\
8.0 & $37 \pm 3$ & $54 \pm 9$ & $56 \pm 5$ & $175 \pm 16$ & 56 \\
11.7 & $20 \pm 4$ & $22 \pm 8$ & $30 \pm 6$ & $63 \pm 12$ & 74 \\
13.2 & $12 \pm 1$ & $17 \pm 7$ & $18 \pm 2$ & $38 \pm 3$ & 68 \\
18.5 & $4 \pm 1$ & $7 \pm 3$ & $6 \pm 2$ & $13 \pm 3$ & 71 \\
\hline \hline
\end{tabular}

${ }^{\mathrm{a}} \sigma\left(\pi^{-} p \rightarrow \omega \Delta^{0}\right)$ derived from the isospin relation $\sigma\left(\pi^{-} p \rightarrow \omega \Delta^{0}\right)=\frac{1}{3} \sigma\left(\pi^{+} p \rightarrow \omega \Delta^{++}\right)$.

${ }^{\mathrm{b}}$ All $\pi^{-} p \rightarrow \omega n$ cross sections derived from data on this reaction and on the reaction $\pi^{+} n \rightarrow \omega p$ (see Refs. 49 and 72).

are within (admittedly large) errors compatible with this equality. ${ }^{37,38}$

\section{Coupling constants}

As was pointed out in Ref. 4 , it is possible to relate the amplitudes of Table III to coupling constants. In one-particle-exchange and Reggeexchange models the amplitudes are proportional to the coupling constants at the baryon vertex. For $K^{-} p \rightarrow M(\Lambda, \Sigma) K$ exchange leads to a ratio of the coupling constants given by $G_{p \Lambda K}^{+} / G_{p\left[0 K^{+}\right.}$ $=(2 f+1) / \sqrt{3}(2 f-1)$ where $f=F /(F+D)$ is the fraction of antisymmetric coupling. This ratio is then equal to the ratio of the amplitudes for $\Lambda$ and $\Sigma^{0}$ production by unnatural-parity exchange. From the amplitudes in Table III and the value of $x$ found above one finds $0.335 \pm 0.012$ for the diquark model and 0.4 for SU(6). Experimentally, the best determination of $f$ stems from the study of the leptonic decays of baryons, which may also be viewed as one-quark transitions with a diquark spectator. Kleinknecht ${ }^{77}$ has summarized these data. He finds $f=0.342 \pm 0.007$ which again agrees perfectly with the diquark value while differing by 8 standard deviations from the SU(6) prediction.

\section{CONCLUSIONS AND DISCUSSIONS}

The diquark hypothesis is capable of explaining a large number of discrepancies between the symmetric-quark-model predictions and the experimental data for strangeness - and chargeexchange two-body processes by introducing only one extra parameter $\left(x=\sin ^{2} \Gamma\right)$. From this

TABLE XIII. Test of diquark relation (14) versus SU(6) relation (15) for $\pi^{-} p \rightarrow \eta^{0}+(n, \Delta)$ and $x \simeq 0.64$ ( $\mu \mathrm{b}$ units). $f_{\mathrm{dq}} \simeq 1.50, f_{\mathrm{SU}(6)}=\frac{25}{8}$.

\begin{tabular}{|c|c|c|c|c|c|}
\hline $\begin{array}{c}p_{\mathrm{lab}} \\
(\mathrm{GeV} / c)\end{array}$ & $\bar{\sigma}(\Delta)=\sigma(\Delta)^{\mathrm{a}}$ & $\bar{\sigma}(n)=\frac{p_{\Delta}^{*}}{p_{n}^{*}} \sigma(n)^{\mathrm{b}}$ & $f_{\mathrm{dq}} \bar{\sigma}(\Delta)$ & $f_{\mathrm{SU}(6)} \bar{\sigma}(\Delta)$ & Ref. \\
\hline 4.0 & $42 \pm 13$ & $88 \pm 12$ & $63 \pm 20$ & $131 \pm 41$ & 53 \\
\hline 4.09 & $62 \pm 10$ & $85 \pm 12$ & $93 \pm 15$ & $194 \pm 31$ & 73 \\
\hline 5.0 & $25 \pm 6^{c}$ & $65 \pm 9$ & $42 \pm 9$ & $88 \pm 19$ & 63 \\
\hline 5.45 & $25 \pm 4$ & $58 \pm 8$ & $38 \pm 6$ & $78 \pm 13$ & 76 \\
\hline 8.0 & $17 \pm 3^{d}$ & $35 \pm 5$ & $26 \pm 5$ & $53 \pm 9$ & 56 \\
\hline 16.0 & $5 \pm 1^{\mathrm{e}}$ & $13 \pm 2$ & $8 \pm 2$ & $17 \pm 4$ & 59 \\
\hline 18.5 & $7 \pm 3$ & $11 \pm 2$ & $11 \pm 5$ & $22 \pm 9$ & 71 \\
\hline
\end{tabular}

${ }^{\mathrm{a}} \sigma\left(\pi^{-} p \rightarrow \eta \Delta^{0}\right)$ derived from the isospin relation $\sigma\left(\pi^{-} p \rightarrow \eta \Delta^{0}\right)=\frac{1}{3} \sigma\left(\pi^{+} p \rightarrow \eta \Delta^{++}\right)$.

${ }^{\mathrm{b}}$ All $\pi^{-} p \rightarrow \eta n$ cross sections derived from the data of Ref. 75 for $t<0.7 \mathrm{GeV}^{2}$.

c The cross section of the backward peak $\left(u^{\prime}<1.0 \mathrm{GeV}^{2}\right)$ has been excluded.

d The cross section is consistent with zero for $t^{\prime}>0.65 \mathrm{GeV}^{2}$.

${ }^{\mathrm{e}} \boldsymbol{t}^{\prime}<1.0 \mathrm{GeV}^{2}$. 
evidence the diquark model emerges as a viable alternative to the symmetric baryon quark model both for parametrizing correlations between the data and for predicting new ones. As stated in the Introduction this conclusion does not necessarily have to imply that the diquark itself exists as an elementary object. Our calculations effectively use only the symmetry requirements resulting from the diquark-quark hypothesis. This hypothesis may be justified either by assuming that the diquark is an elementary object or by endowing the spatial part of the baryon wave function with the property that one quark be sufficiently far removed from the other two. The latter justification is less far-reaching but sufficient and therefore is (in our opinion) more likely. Our conclusions raise the question not only of the validity of this diquark model outside the field of quasi-two-body reactions considered here but also of the extent of its direct geometrical validity. Before briefly considering these questions it is perhaps worthwhile to state that the diquark vs symmetric baryon picture does not have to be strictly either/or. It is quite possible that the baryon spends a fraction of its time in a three-quark configuration and the remaining time in a quark-diquark configuration and that peripheral quasi-two-body reactions are just preferentially initiated from a baryon which is in the diquark-quark configuration.

The first field one is led to think about is the static properties of the baryons as they result from the diquark-quark hypothesis. As stated in the Introduction the diquark relations between static hadron properties are in general indistinguishable from the pure SU(6) ones; their agreement with the experimental data can be qualified as varying from reasonable to poor.

Interesting manifestations of a possible diquark structure inside the nucleons have been discussed by Pavkovic ${ }^{78}$ and Franklin. ${ }^{79}$ Both authors looked into the well-known discrepancy between the $\mathrm{SU}(6)$ predictions for the nucleon structure functions $\nu W_{2}$ and the SLAC data. SU(6) predicts the ratio of $\left(\nu W_{2}\right)_{n}$ to $\left(\nu W_{2}\right)_{p}$ to be a constant (equal to $\left.\frac{2}{3}\right)$; the data, however, show a behavior varying between 0.9 and 0.3 for $\zeta$ (the Bjorken-Drell scaling variable) varying form 0 to 1 . Pavković concluded that it is possible to explain these results on the basis of a baryon with quark-diquark symmetry. ${ }^{80}$ Franklin did not use a diquark model as input but came to the conclusion that a study of the nucleon structure functions strongly suggests that no SU(6) symmetry can be imposed if one wants to explain the data; only a baryon treated as two uncoupled oscillators, a diquark oscillator, and a quark-diquark oscillator gave a good understanding of the proton/neutron structure functions. The latter approach is interesting as it seems to obtain a geometrically defined diquark-quark substructure in the nucleon starting from nonsymmetry arguments. Earlier Ono had shown that the momentum transfer distributions of (inelastic) electroproduction processes of the type $e+p \rightarrow e+$ isobar could be explained using the baryon model of a quark and diquark held together by a potential of the Wood-Saxon type. ${ }^{81}$

Several dynamical models have obtained diquark clustering from quark-quark interactions within the baryon. Models by Mitra ${ }^{82}$ and Capps ${ }^{83}$ using quark-diquark exchange forces generated by the orbital motion of the quarks in addition to a shortrange quark-quark force have been shown to lead to the minimal baryon spectrum. More recently Eguchi $^{84}$ showed that the (rotating) string model relation between energy and angular momentum $\left(E^{2} \sim l\right)$ implies that either a quark-diquark or a linear three-quark structure is the energetically favored configuration for the baryon valence quarks. The resulting baryon spectrum is again the minimal one. Johnson and Thorn ${ }^{85}$ showed that in the MIT bag model stringlike (linear) baryon structures are interpretable as deformed bags containing (colored) quarks and gluons. Both approaches correlate a possible cigarlike shape of baryons (and the resulting clustering of quarks inside the baryon) primarily with baryons in high angular momentum states. The latter authors point out, however, that calculations indicate that even the ground-state hadrons may themselves be deformed. In any case our experimental evidence does not relate to all possible quark-string orientations; the peripheral nature of the interactions studied could effectively act as a filter selecting those initial states where the quarkdiquark string stands perpendicular to the incident directions.

Cutkosky et $a l .^{86}$ have shown that a baryon model with linear string potentials between the valence quarks (along with a strong attractive two-body and a smaller repulsive three-body component) could reproduce the known baryon spectra and in particular accommodate an experimentally desired low-lying $\left(56,1^{-}\right)$multiplet; geometrically such a potential would imply the existence of a substantial degree of diquark clustering.

Recently Preparata and Szegö ${ }^{87}$ studying a bag-type model and using essentially only geometry arguments found that their spatial wave equations could only be solved if nature would "help" by suppressing the internal geometrical degrees of freedom for the quarks inside the baryon to just three. The spatial configuration of a quark-diquark corresponds to just such a 
suppression.

A final remark concerns the significance of possible diquark substructure inside the baryons. It is not limited to the fact that it would shed light on the dynamics of $q q q$ states itself; diquarks could also imply the existence of exotic mesons of the type $(q q) \pm(\overline{q q}) \cdot{ }^{85,88}$

In conclusion, we have found that the diquark model, with just one extra parameter, can explain a large amount of two-body scattering data which are in violent disagreement with the SU(6)symmetric quark model. It will be interesting to see how this model, of which we have essentially used only the symmetry, can be further explained by more fundamental dynamical models.

\section{ACKNOWLEDGMENTS}

We would like to thank the other members of the Amsterdam-CERN-Nijmegen-Oxford collaboration for their interest. In particular, we thank Professor J. C. Kluyver for the valuable suggestion which led to this investigation and for several lively and instructive discussions during the course thereof. We also acknowledge the help of the Amsterdam group in collecting much of the data presented in our tables. Discussions with Dr. Pennington, Dr. Wolters, and Dr. Massaro have been helpful. The work of the Nijmegen authors was partially supported by the joint research program of the Dutch organizations for fundamental research FOM and ZWO.
*On leave from Silesian University, Katowice, Poland. †Visiting scientist from the Tata Institute of Fundamental Research, Bombay, India.

${ }^{1} J$. Kuti and V. F. Weisskopf, Phys. Rev. D 4, 3418

(1971); O. Nachtmann, Nucl. Phys. B38, 397 (1972).

${ }^{2} \mathrm{H}$. J. Lipkin, in Proceedings of the Fifth Coral Gables Conference on Symmetry Principles at High Energies, University of Miami, 1968, edited by A. Perlmutter, G. Anges Hurst, and B. Kurşunoğlu (Benjamin, New York, 1968), p. 261; H. J. Lipkin, Phys. Rep. 8C, 173 (1973).

${ }^{3} \mathrm{E}$. Hirsch et al., Phys. Lett. 36B, 139 (1971).

${ }^{4} J$. C. Kluyver et al., Nucl. Phys. B140, 141 (1978); G.

G. G. Massaro, thesis, University of Amsterdam, 1978 (unpublished).

${ }^{5}$ H. J. Lipkin, Nucl. Phys. B7, 321 (1968); G. Alexander et al., Phys. Rev. Lett. 17, 412 (1966).

${ }^{6}$ M. J. Losty et al., Nucl. Phys. B133, 38 (1978).

${ }^{7}$ E. Hirsch, U. Karshon, and H. J. Lipkin, Phys. Lett. 36B, 385 (1971).

${ }^{8}$ M. ZraYek, University of Nijmegen Internal Report No. HEN-172, 1978 (unpublished).

${ }^{9}$ D. B. Lichtenberg and L. J. Tassie, Phys. Rev. 155, 1601 (1967).

${ }^{10}$ P. D. De Souza and D. B. Lichtenberg, Phys. Rev. 161, 1513 (1967); D. B. Lichtenberg, Nuovo Cimento 49, 435 (1967); P. J. Keleman et al., Phys. Rev. 167, 1535 (1968).

${ }^{11}$ E. M. Levin and L. L. Frankfurt, Zh. Eksp. Teor. Fiz. Pis'ma Red. 2, 105 (1965) [JETP Lett. 2, 65 (1965)]; H. J. Lipkin and F. Scheck, Phys. Rev. Lett. 16, 71 (1966); J. J. J. Kokkedee and L. Van Hove, Nuovo Cimento 42, 711 (1966).

${ }^{12}$ D. B. Lichtenberg, Phys. Rev. 178, 2197 (1969).

${ }^{13}$ J. Carroll, D. B. Lichtenberg, and J. Franklin, Phys. Rev. 174, 1681 (1968).

${ }^{14} \mathrm{~J}$. J. de Swart, Rev. Mod. Phys. 35, 916 (1963).

${ }^{15}$ In baryon-baryon scattering elastic diquark-diquark amplitudes should play a role; however, there are a large number of independent spin amplitudes for such processes (e.g. proton-proton elastic scattering involves 29 elastic diquark-diquark amplitudes) and this makes a comparison with experiment practically im.possible. In antibaryon-baryon scattering diquark annihilation-creation amplitudes would be expected to contribute but again too many amplitudes are involved for a meaningful comparison with the available data. Elastic (as well as inelastic) antiquark-diquark amplitudes would mediate backward scattering in reactions (1) and (2); once more, too many amplitudes are involved (even if the inelastic ones are ignored).

${ }^{16} \mathrm{~J}$. P. Ader et al., Nuovo Cimento 56A, 952 (1968).

${ }^{17} \mathrm{~A}$. Bialas and K. Zalewski, Phys. Lett. 26B, 180 (1968); A. Białas and K. Zalewski, Nucl. Phys. B6, 465 (1968).

${ }^{18} \mathrm{~K}$. Böckmann et al., Phys. Lett. 28B, 72 (1963); M. Aderholz et al., Nucl. Phys. $\overline{\mathrm{B} 8}, 503$ (1968); A. Kotanski and K. Zalewski, ibid. B13, 119 (1969); H. Friedman and R. R. Ross, Phys. Rev. Lett. 22, 152 (1969); W. De Baere et al., Nuovo Cimento 56, 397 (1969); B. Haber et al., Nucl. Phys. B17, $2 \overline{89}$ (1970).

${ }^{19}$ Other examples for reactions (6) and (7), again with $M=1^{-}$, are the following unnatural-parity exchange relations between the polarizations of the baryons (see Ref. 4):

$P_{U}^{m}(\Lambda)=P_{U}^{m}(\Sigma)=\frac{2 \sqrt{3}}{\sqrt{5}} P_{U}^{m}(Y)$

and

$P_{U}^{m}(n)=\frac{2 \sqrt{3}}{\sqrt{5}} P_{U}^{m}(\Delta)$.

${ }^{20}$ H. J. Lipkin and F. Scheck, Phys. Rev. Lett. 18, 347 (1967).

${ }^{21}$ We use a polarization vector $\vec{P}$ defined by

$$
\vec{P}=\left[\frac{3}{2 s^{2}(s+1)}\right]^{1 / 2}\langle\overrightarrow{\mathrm{s}}\rangle,
$$

where $s$ is the spin of the particle considered.

${ }^{22}$ T. Hofmokl and M. Szeptycka, Nucl. Phys. B13, 53 (1969).

${ }^{23}$ S. Meshkov, G. A. Snow, and G. B. Yodh, Phys. Rev. Lett. 12, 87 (1964).

${ }^{24}$ G. H. Trilling, Nucl. Phys. B40, 13 (1972).

${ }^{25}$ Using the reactions $K^{-} p \rightarrow \overline{\mathrm{A} \phi}$ and $K^{-} p \rightarrow Y \phi$ the value $x=0.60 \pm 0.02$ is obtained.

${ }^{26}$ J. C. Scheuer et al., Nucl. Phys. B33, 61 (1971).

${ }^{27} \mathrm{U}$. Karshon et al., Nuc1. Phys. B29, 557 (1971).

${ }^{28}$ M. Aguilar-Benitez et al., Phys. Rev. D 6, 29 (1972).

${ }^{29} \mathrm{~B}$. Chaurand et al., Nucl. Phys. B117, 1 (1976). 
${ }^{30}$ S. O. Holmgren et al., Nucl. Phys. B119, 261 (1977);

G. G. G. Massaro et al ., Phys: Lett. $66 \mathrm{~B}, 385$ (1977);

F. Marzano et al., Nucl. Phys. B123, 203 (1977).

${ }^{31}$ M. Aguilar-Benitez et al., Nucl. Phys. B124, 189 (1977).

${ }^{32} \mathrm{D}$. Yaffe et al., Nucl. Phys. B75, 365 (1974).

${ }^{33}$ D. J. Crennell et al., Phys. Rev. D 6, 1220 (1972).

${ }^{34}$ In addition, the $Y$ polarization has to be zero. Experimentally there is a small nonzero polarization of the $Y$ for $t^{\prime}<0.2 \mathrm{GeV}^{2}$ which may be related to the small $I=\frac{3}{2}$ exchange amplitude which has been found necessary to describe the reaction $K^{-} p \rightarrow \pi^{-} Y^{+}$at $4.2 \mathrm{GeV} / c$ (Ref. 30).

${ }^{35} \mathrm{~K}$. S. Foley et al., Phys. Rev. D 8, 27 (1973).

${ }^{36}$ A. M. Boyarski et al., Phys. Lett. 34B, 547 (1971). The data for $\gamma p \rightarrow K^{+} \Lambda$ and $K^{+} \Sigma^{0}$ are from an experiment at $11 \mathrm{GeV}$; the $\gamma p \rightarrow K^{+} Y$ data are from experiments between 2 and $5.8 \mathrm{GeV}$. It was assumed that the cross sections scale as $k^{-2}$.

${ }^{37} \mathrm{H}$. G. J. M. Tiecke, thesis, University of Nijmegen, 1974 (unpublished).

${ }^{38}$ W. J. Metzger, University of Nijmegen Internal Report No. HEN-166, 1977 (unpublished).

${ }^{39}$ F. Marzano et al ., Phys. Lett. 68B, 292 (1977); J. J. Engelen (private communication).

${ }^{40} \mathrm{~J}$. Gallivan et al., Nucl. Phys. B117, 269 (1976).

${ }^{41}$ M. G. D. Gilchriese et al., Phys. Rev. Lett. 40, 6 (1978).

${ }^{42}$ G. W. Brandenburg et al., Phys. Rev. D 15, 617 (1977).

${ }^{43} \mathrm{~K}$. J. Foley et al., Phys. Rev. D 9, $42(1 \overline{974})$.

${ }^{44}$ I. Butterworth et al., Phys. Rev. Lett. 15, 734 (1965);

F. Bomse et al., Phys. Rev. 158, 1298 (1967).

${ }^{45}$ G. Bassompierre et al., Nucl. Phys. B16, 125 (1970).

${ }^{46}$ Y. Goldschmidt-Clermont et al., Phys. Lett. 27B, 602 (1968).

${ }^{47}$ E. Bracci et al., CERN Report No. CERN/HERA 72-2 (unpublished).

${ }^{48} \mathrm{M}$. Haguenauer et al., Phys. Lett. 37B, 538 (1971);

K. W. J. Barnham et al., Nucl. Phys. B28, 171 (1971).

${ }^{49}$ J. A. J. Matthews et al., Phys. Rev. Lett. 26, 400 (1971).

${ }^{50}$ S. U. Chung et al., Phys. Rev. D 12, 693 (1975).

${ }^{51} \mathrm{P}$. Sonderegger et al., Phys. Lett. 20, 75 (1966).

${ }^{52}$ B. Haber et al., Phys. Rev. D 11, $\overline{495}$ (1975).

${ }^{53}$ M. Aderholz et al., Phys. Rev. 138, B897 (1965); private communication from N. $\overline{\mathrm{Sch} m i t z}$ to the Particle Data Group, 1969, as reported in LBL Report No. LBL-53 (unpublished).

${ }^{54}$ D. J. Schotanus et al., Nucl. Phys. B22, 45 (1970).

${ }^{55}$ T. J. Bloodworth et al., Nucl. Phys. $\overline{\mathrm{B} 81}, 231$ (1974).

${ }^{56} \mathrm{M}$. Aderholz et al., Nucl. Phys. B8, 45 (1968).

${ }^{57} \mathrm{D}$. Evans et al., Nuovo Cimento $\overline{16 \mathrm{~A}}, 299$ (1973).

${ }^{58} \mathrm{~J}$. H. Scharenguivel et al., Nucl. Phys. B36, 363 (1972).

${ }^{59} \mathrm{R}$. Honecker et al., Nucl. Phys. B131, 189 (1977).

${ }^{60} \mathrm{~B}$. Haber et al., Phys. Rev. D 10, 1387 (1974).

${ }^{61}$ L. Bondár et d., Nuovo Cimento 31,729 (1964); R. L. Eis- ner et al., Phys. Rev. 164, 1699 (1967); D. J. Crennell et al., Phys. Rev. Lett. 27, 1674 (1971); J. A. J. Matthews et al., Nucl. Phys. B32, 366 (1971); K. Takahashi et al., Phys. Rev. D $\underline{6}, 1266$ (1972); C. Caso et al ., Nuovo Cimento 62A, 755 (1969); B. D. Hyams et al., Nuc1. Phys. B7, 1 (1968); J. Ballam et al., Phys. Lett. 31B, 489 (1970); J. Bartsch et al., Nucl. Phys. B46, 46 (1972).

${ }^{62} \mathrm{D}$. Brown et al., Phys. Rev. D1, 3053 (1970). ${ }^{63} \mathrm{C}$. L. Pols et al., Nucl. Phys. B25, 109 (1970). ${ }^{64}$ Y. Eisenberg et al., Phys. Lett. 48B, 354 (1974). ${ }^{65}$ I. J. Bloodworth et al., Nucl. Phys. B35, 79 (1971). ${ }^{66}$ R. O. Maddock et al., Nuovo Cimento 5A, 433 (1971). ${ }^{67} \mathrm{~J}$. A. Gaidos et al., Phys. Rev. D 1, 3190 (1970). ${ }^{68} \mathrm{~J}$. A. Gaidos et al., Nucl. Phys. B72, 253 (1974). ${ }^{69} \mathrm{~J}$. Ballam et al., Phys. Rev. D 4, 1946 (1971).

${ }^{70} \mathrm{M}$. Deutschmann et al., Nucl. Phys. B99, 397 (1975).

${ }^{71}$ N. N. Biswas et al., Phys. Rev. D 2, 2529 (1970).

${ }^{72} \mathrm{G}$. C. Benson et al., Phys. Rev. Lett. 22, 1074 (1969); M. J. Emms et al., Nucl. Phys. B98, 1 (1975); G. S. Abrams et al., Phys. Rev. Lett. 23, 673 (1969); L. E. Holloway et al., Phys. Rev. D 8,2814 (1973); N. Armenise et al., Nuovo Cimento 65A, 637 (1970); M. S. Farber et al., Nucl. Phys. B29, 237 (1971); J. C. Anderson et al., Phys. Lett. 45B, 165 (1973); K. Paler et al., Lett. Nuovo Cimento $\underline{4}, 745$ (1972); V. N. Bolotov et al., Yad. Fiz. 21, 316 (1975) [Sov. J. Nucl. Phys. 21, 166 (1975)].

${ }^{73} \mathrm{D}$. G. Fong et al., Phys. Rev. D 9 , 3015 (1974).

${ }^{74}$ D. Evans et al., Nucl. Phys. B51, 205 (1973).

${ }^{75}$ O. Guisan et al., Phys. Lett. 18,200 (1965); V. N. Bolotov et al., Nucl. Phys. B73, 387 (1974).

${ }^{76}$ I. J. Bloodworth et al., Nucl. Phys. B39, 525 (1972).

${ }^{77} \mathrm{~K}$. Kleinknecht, in Proceedings of the XVII International Conference on High Energy Physics, London, 1974, edited by J. R. Smith (Rutherford Laboratory, Didcot, Berkshire, England, 1974), Vol. III, p. 26.

${ }^{78}$ M. I. Pavkovic, Phys. Rev. D 13, 2128 (1976).

${ }^{79}$ J. Franklin, Phys. Rev. D 16, 21 (1977).

${ }^{80}$ The $x$ values used by Pavkovic are not well defined; they lie in the range 0.3-0.7 and are thus compatible with ours.

${ }^{81}$ S. Ono, Phys. Rev. D $\underline{9}, 2005$ (1974).

${ }^{82}$ A. N. Mitra, Ann. Phys. (N.Y.) 43, 126 (1967); Nucl. Phys. B5, 308 (1968); Nuovo Cimento 56, 1164 (1968).

${ }^{83}$ R. H. Capps, Phys. Rev. Lett. 33, 1637 (1974).

${ }^{84}$ T. Eguchi, Phys. Lett. 59B, 457 (1975).

${ }^{85} \mathrm{~K}$. Johnson and C. B. Thorn, Phys. Rev. D $\underline{13}, 1934$ (1976).

${ }^{86}$ R. E. Cutkosky et al., Phys. Rev. Lett. 37, 645 (1976); R. E. Cutkosky and R. E. Hendrick, Phys. Rev. D 16, 2902 (1977).

${ }^{87}$ G. Preparata and K. Szegö, Phys. Lett. 68B, 239 (1977).

${ }^{88}$ R. L. Jaffe, Phys. Rev. D $\underline{17}, 1444$ (1978). 